\title{
GRACE/SUSY \\ Automatic Generation of Tree Amplitudes in the Minimal Supersymmetric Standard Model
}

\author{
J. Fujimoto $^{\text {a }}$ T. Ishikawa ${ }^{\text {a }}$ M. Jimbo ${ }^{b}$ T. Kaneko ${ }^{\text {a }}$ K. Kato ${ }^{c}$ \\ S. Kawabata ${ }^{a}$ K. Kon ${ }^{d}$ M. Kuroda ${ }^{e}$ Y. Kurihara ${ }^{a}$ Y. Shimizu ${ }^{a}$ \\ H. Tanaka ${ }^{\mathrm{f}}$ \\ ${ }^{a}$ KEK, Oho, Tsukuba, Ibaraki 305-0801 Japan \\ b Tokyo Management College, Ichikawa, Chiba 272-0001, Japan \\ ${ }^{\mathrm{c}}$ Kogakuin University, Nishi-Shinjuku, Tokyo 163-867\%, Japan \\ d Seikei University, Musashino, Tokyo 180-8633, Japan \\ e Meijigakuin University, Totsuka, Yokohama 244-8539, Japan \\ ${ }^{\mathrm{f}}$ Rikkyo University, Nishi-Ikebukuro, Tokyo 171-8501, Japan
}

\begin{abstract}
GRACE/SUSY is a program package for generating the tree-level amplitude and evaluating the corresponding cross section of processes of the minimal supersymmetric extension of the standard model (MSSM). The Higgs potential adopted in the system, however, is assumed to have a more general form indicated by the two-Higgsdoublet model. This system is an extension of GRACE for the standard model(SM) of the electroweak and strong interactions. For a given MSSM process the Feynman graphs and amplitudes at tree-level are automatically created. The Monte-Carlo phase space integration by means of BASES gives the total and differential cross sections. When combined with SPRING, an event generator, the program package provides us with the simulation of the SUSY particle productions.
\end{abstract}

\section{Introduction}

It is widely expected that the supersymmetric (SUSY) extension of the standard model(SM) of particle physics [1] is one of the most promising approaches that could resolve the problems remaining in the standard model. In particular, the minimal supersymmetric extension of the standard model (MSSM) has been extensively studied in the last decade, because it has the simplest 
structure containing the least number of particles, yet it is complex enough to allow the most essential features characteristic in any theory of SUSY.

Over these ten years we have been working with the automatic computation of high energy physics processes. The system for the computation of the SM, GRACE, has been published in [2]. In order to be able to handle SUSY processes, we have made several necessary modifications and extension of the system $[3,4]$. First we have installed the new interaction vertices according to the MSSM lagrangian allowing an extension to a more general effective Higgs potential (section 2.4). Second we have modified the program so that we can deal with the fermion number non-conserving vertices and Majorana particles, both of which are inherent to any SUSY theory.

Using this extended version of GRACE/SUSY, we have published a package of event-generator, SUSY23, which contains 23 specific SUSY processes for $e^{+} e^{-} \rightarrow$ 2-body and 3-body [5].

Since then we have made extensive checks of the system. In this paper we present the detailed description of the program system which enables us to compute automatically the cross section of any MSSM tree process, and explain the structure of GRACE/SUSY together with instructions for how to use it.

Program pacakge GRACE/SUSY (GRACE v2.2.0) is available form http://minami-home.kek.jp/.

\section{Theoretical framework of the MSSM}

\subsection{Basic lagrangian}

The basic lagrangian of the MSSM in its full form has been given in several papers[6-8]. In this work we use the convention adopted in ref.[8], where the positively (negatively) charged charginos are considered as fermion (antifermion). The full form of the MSSM lagrangian is too lengthy to be reproduced here. Readers should refer to the original work [8]. We show only the soft SUSY-breaking terms in order to define the SUSY breaking parameters.

$$
\begin{aligned}
\mathcal{L}_{\text {soft }}= & -\mu \int d^{2} \theta \Phi_{\mathbf{H}_{\mathbf{1}}} \Phi_{\mathbf{H}_{\mathbf{2}}}+\text { h.c. } \\
& -\frac{1}{2} M_{1} \lambda \lambda-\frac{1}{2} M_{2} \lambda^{a} \lambda^{a}-\frac{1}{2} M_{3} \tilde{g}^{\alpha} \tilde{g}^{\alpha}+h . c .
\end{aligned}
$$




$$
\begin{aligned}
& -\tilde{m}_{1}^{2} \mathbf{H}_{1}^{*} \mathbf{H}_{1}-\tilde{m}_{2}^{2} \mathbf{H}_{2}^{*} \mathbf{H}_{2}-\left(\tilde{m}_{12}^{2} \mathbf{H}_{1}\left(\begin{array}{cc}
0 & 1 \\
-1 & 0
\end{array}\right) \mathbf{H}_{2}+\text { h.c. }\right) \\
& -\frac{\sqrt{2} m_{u}}{v_{2}} A_{u} \mathbf{H}_{2} \mathbf{A}\left(q_{L}\right) A\left(u_{R}\right)+\frac{\sqrt{2} m_{d}}{v_{1}} A_{d} \mathbf{H}_{1} \mathbf{A}\left(q_{L}\right) A\left(d_{R}\right)+h . c . \\
& +\frac{\sqrt{2} m_{e}}{v_{1}} A_{e} \mathbf{H}_{1} \mathbf{A}\left(\ell_{L}\right) A\left(e_{R}\right)+h . c \\
& -\sum_{f}\left[\tilde{m}_{\tilde{f}_{L}}^{2} \tilde{f}_{L}^{*} \tilde{f}_{L}+\tilde{m}_{\tilde{f}_{R}}^{2} \tilde{f}_{R}^{*} \tilde{f}_{R}\right] .
\end{aligned}
$$

In the first line $\Phi_{\mathbf{H}_{1}}$ and $\Phi_{\mathbf{H}_{2}}$ stand for the iso-doublet Higgs superfields with hypercharge $Y=-1$ and $Y=+1$ respectively, and in the second line $\lambda, \lambda^{a}$ and $\tilde{g}^{\alpha}$ are the gaugino fields for $U(1), S U(2)$ and $S U(3)$ gauge symmetries, respectively. $\mathbf{H}_{i}$ appearing in the third to fifth line are the Higgs doublets which are decomposed as

$$
\begin{aligned}
& \mathbf{H}_{1} \equiv\left(\begin{array}{c}
H_{1}^{0} \\
H_{1}^{-}
\end{array}\right)=\left(\begin{array}{c}
\left(v_{1}+\phi_{1}^{0}-i \chi_{1}^{0}\right) / \sqrt{2} \\
-\phi_{1}^{-}
\end{array}\right), \\
& \mathbf{H}_{2} \equiv\left(\begin{array}{c}
H_{2}^{+} \\
H_{2}^{0}
\end{array}\right)=\left(\begin{array}{c}
\phi_{2}^{+} \\
\left(v_{2}+\phi_{2}^{0}+i \chi_{2}^{0}\right) / \sqrt{2}
\end{array}\right) .
\end{aligned}
$$

The scalar fields $\mathbf{A}\left(q_{L}\right), A\left(u_{R}\right)$, etc. represent the sfermion fields, the superpartners of the corresponding fermion fields $\left(\begin{array}{c}u_{L} \\ d_{L}\end{array}\right)$ and $\bar{u}_{R}$, respectively,

$$
\mathbf{A}\left(q_{L}\right)=\left(\begin{array}{c}
\tilde{u}_{L} \\
\tilde{d}_{L}
\end{array}\right), \quad A\left(u_{R}\right)=\tilde{u}_{R}^{*}, \quad A\left(d_{R}\right)=\tilde{d}_{R}^{*} .
$$

Although the so-called $R$-parity violating interactions [9] are not included in this lagrangian and hence in GRACE/SUSY, users can implement them in the system by coding the necessary vertex functions etc. in appropriate subprograms.

\subsection{Particle content}

The MSSM consists of superfields containing the known fermions or bosons as their component fields, and two Higgs superfields. Note that in contrast to the SM, the MSSM includes three neutral Higgs particles and a pair of charged Higgs particles. The mass eigenstates of the CP-even neutral Higgs states are defined by

$$
\left(\begin{array}{l}
H^{0} \\
h^{0}
\end{array}\right)=\left(\begin{array}{cc}
\cos \alpha & \sin \alpha \\
-\sin \alpha & \cos \alpha
\end{array}\right)\left(\begin{array}{c}
\phi_{1}^{0} \\
\phi_{2}^{0}
\end{array}\right)
$$


where the rotation angle $\alpha$ is determined in such a way that the Higgs mass matrix becomes diagonal. Since any of the known particles has its superpartner, the number of the elementary particles in the MSSM is more than double the number of particles appearing in the SM. In fact, for each known fermion $f$ (except for neutrinos), there are two kinds of sfermions, the lefthanded sfermion $\tilde{f}_{L}$ and the right-handed sfermion $\tilde{f}_{R}$. The mass eigenstates of sfermion $\tilde{f}_{i}$ with mass $m_{\tilde{f}_{i}}\left(m_{\tilde{f}_{1}}<m_{\tilde{f}_{2}}\right)$ is defined by the orthogonal matrix

$$
\left(\begin{array}{l}
\tilde{f}_{1} \\
\tilde{f}_{2}
\end{array}\right)=\left(\begin{array}{cc}
\cos \theta_{f} & \sin \theta_{f} \\
-\sin \theta_{f} & \cos \theta_{f}
\end{array}\right)\left(\begin{array}{c}
\tilde{f}_{L} \\
\tilde{f}_{R}
\end{array}\right), \quad f=e, u, d, \cdots
$$

where the rotation angle is determined by the mass diagonalization condition.

For each gauge boson there is a gaugino, and to each Higgs particle corresponds a higgsino. Two neutral gauginos, $\lambda$ and $\lambda^{0}$, and two neutral higgsinos, $\tilde{H}_{1}^{0}$ and $\tilde{H}_{2}^{0}$, are combined to form four Majorana fermions called neutralinos, $\tilde{\chi}_{i}^{0} \equiv\left(\begin{array}{c}\lambda_{i} \\ \lambda_{i}\end{array}\right)$. The two-component spinors $\lambda_{i}(i=1 \sim 4)$ are linear combinations of $\lambda, \lambda^{0}, \tilde{H}_{1}^{0}$ and $\tilde{H}_{2}^{0}$,

$$
\tilde{\lambda}_{i}=\eta_{i}\left(\mathcal{O}_{N}\right)_{i j} \chi_{j}, \quad i=1 \sim 4
$$

where $\chi_{j}=\left(\lambda, \lambda^{0}, \tilde{H}_{1}^{0}, \tilde{H}_{2}^{0}\right)$, and masses are ordered as $m_{\tilde{\chi}_{1}^{0}} \leq m_{\tilde{\chi}_{2}^{0}} \leq m_{\tilde{\chi}_{3}^{0}} \leq$ $m_{\tilde{\chi}_{4}^{0}}$. The sign factor $\eta_{i}$ takes the value of +1 or $+i$, and $\mathcal{O}_{N}$ is the orthogonal matrix which diagonalizes the mass matrix of the $\left(\lambda, \lambda^{0}, \tilde{H}_{1}^{0}, \tilde{H}_{2}^{0}\right)$ system.

The other neutral gauginos of the strong interaction, gluinos, remain unmixed with other particles.

Corresponding to the charged Higgs particles $H^{ \pm}$and charged gauge bosons $W^{ \pm}$, there are two charged higgsinos, $\tilde{H}_{1}^{-}$and $\tilde{H}_{2}^{+}$, and a pair of charged gauginos (wino), $\lambda^{ \pm}$. Two charged fermions called charginos, $\tilde{\chi}_{i}^{+}=\left(\begin{array}{c}\overline{\lambda_{i R}^{-}} \\ \lambda_{i L}^{+}\end{array}\right)$, are linear combination of these particles,

$$
\begin{aligned}
& \left(\begin{array}{l}
\lambda_{1 R}^{-} \\
\lambda_{2 R}^{-}
\end{array}\right)=\left(\begin{array}{cc}
\cos \phi_{R} & \sin \phi_{R} \\
-\sin \phi_{R} & \cos \phi_{R}
\end{array}\right)\left(\begin{array}{c}
\lambda^{-} \\
\tilde{H}_{1}^{-}
\end{array}\right), \\
& \left(\begin{array}{c}
\lambda_{1 L}^{+} \\
\lambda_{2 L}^{+}
\end{array}\right)=\left(\begin{array}{cc}
1 & 0 \\
0 & \epsilon_{L}
\end{array}\right)\left(\begin{array}{cc}
\cos \phi_{L} & \sin \phi_{L} \\
-\sin \phi_{L} & \cos \phi_{L}
\end{array}\right)\left(\begin{array}{c}
\lambda^{+} \\
\tilde{H}_{2}^{+}
\end{array}\right) .
\end{aligned}
$$

The mixing angle $\phi_{R}, \phi_{L}$ and the sign factor $\epsilon_{L}$ are determined so that their masses satisfy the ordering $0 \leq m_{\tilde{\chi}_{1}^{ \pm}} \leq m_{\tilde{\chi}_{2}^{ \pm}} \square$.

1 Note that the value of the mixing angles $\phi_{L}$ and $\phi_{R}$ become unstable when $\tan \beta=$ $-\mu / M_{2}$ or $\cot \beta=-\mu / M_{2}$. If this happens, $\tan \phi_{L}$ or $\tan \phi_{R}$ become $\pm \infty$. 
Together with the particles appearing in the SM, these SUSY-particles constitute the ingredient of the MSSM. The list of the particles and their coding names is given in Appendix A.

\subsection{Basic parameters of the MSSM}

The basic parameters of the MSSM come in when the lagrangian is expressed in terms of superfields [8]. They are

\begin{tabular}{|l|l|}
\hline coupling constants & $g, g^{\prime}, g_{s}, \mu$ \\
gaugino mass parameters & $M_{1}, M_{2}, M_{3}$ \\
fermion masses (for each generation) & $m_{e}, m_{u}, m_{d}$ \\
Higgs mass parameters & $\tilde{m}_{1}, \tilde{m}_{2}, \tilde{m}_{12}$ \\
trilinear couplings(for each generation) & $A_{e}, A_{u}, A_{d}$ \\
sfermion mass terms(for each generation) & $\tilde{m}_{\tilde{e}_{L}}, \tilde{m}_{\tilde{e}_{R}}, \tilde{m}_{\tilde{u}_{L}}, \tilde{m}_{\tilde{u}_{R}}, \tilde{m}_{\tilde{d}_{R}}$ \\
\hline
\end{tabular}

The vacuum expectation values $v_{1}$ and $v_{2}$ are determined at the minimum of the Higgs potential and they are expressed in terms of these basic parameters. Assuming massless neutrinos, there are in all 4 coupling constants, $3 N_{G}$ fermion masses, $6+8 N_{G}$ soft supersymmetry breaking parameters, where $N_{G}$ stands for the number of fermion generations.

\subsection{The MSSM parameters in GRACE/SUSY}

As alternatives to ten MSSM parameters for the coupling constants and gaugino and Higgs masses shown above, we can use in GRACE/SUSY the following set of ten parameters as inputs,

$$
\alpha_{e}, \alpha_{s}, M_{W}, M_{Z}, \tan \beta, M_{A^{0}}, \mu, M_{1}, M_{2}, M_{3}
$$

Unless specified explicitly by the user (see Appendix B), $M_{1}$ and $M_{3}$ are computed from $M_{2}$ by the following unification conditions

$$
\begin{aligned}
& M_{1}=\frac{5}{3} \tan ^{2} \theta_{W} M_{2} \\
& M_{3}=\frac{g_{s}^{2}}{e^{2}} \sin ^{2} \theta_{W} M_{2} .
\end{aligned}
$$


Concerning the eight sfermion mass parameters in each generation, we use three mixing angles, and two up-type squark masses, the lighter down-type squark mass, and two down-type slepton masses as independent input parameters. Due to the $S U(2)_{L}$ invariance of the $\mathcal{L}_{\text {soft }}$, the heavier down-type squark mass and the sneutrino mass are given as the solution of the following two equations (shown here only for the third generation sfermions),

$$
\begin{gathered}
\sin ^{2} \theta_{b} m_{\tilde{b}_{2}}^{2}=\cos ^{2} \theta_{t} m_{\tilde{t}_{1}}^{2}+\sin ^{2} \theta_{t} m_{\tilde{t}_{2}}^{2}-\cos ^{2} \theta_{b} m_{\tilde{b}_{1}}^{2}+m_{b}^{2}-m_{t}^{2}-M_{W}^{2} \cos 2 \beta \\
m_{\tilde{\nu}_{\tau}}^{2}=\cos ^{2} \theta_{\tau} m_{\tilde{\tau}_{1}}^{2}+\sin ^{2} \theta_{\tau} m_{\tilde{\tau}_{2}}^{2}-m_{\tau}^{2}+M_{W}^{2} \cos 2 \beta
\end{gathered}
$$

Since we restrict our consideration only to the CP conserving processes, all the parameters are real. In particular, all the physical masses used as the input are real and positive. The allowed range of the SUSY parameters used in GRACE/SUSY whose values are not yet fixed by experiments are as follows ${ }^{2}$

$$
\begin{aligned}
& -\infty<\mu<+\infty, \quad 0 \leq \tan \beta, \quad 0 \leq M_{1}, M_{2}, M_{3}, M_{A^{0}}, \\
& 0 \leq m_{\tilde{f}_{1}} \leq m_{\tilde{f}_{2}}, \quad-\frac{\pi}{2}<\theta_{f} \leq+\frac{\pi}{2} .
\end{aligned}
$$

The CP-even Higgs, charginos and neutralinos are numbered as

$$
M_{h^{0}}<M_{H^{0}}, \quad m_{\tilde{\chi}_{1}^{ \pm}}<m_{\tilde{\chi}_{2}^{ \pm}}, \quad m_{\tilde{\chi}_{1}^{0}}<m_{\tilde{\chi}_{2}^{0}}<m_{\tilde{\chi}_{3}^{0}}<m_{\tilde{\chi}_{4}^{0}} .
$$

The basic parameters of the MSSM are expressed in terms of these GRACE/SUSY variables given in (2.8) as follows,

$$
\begin{aligned}
& g=e \sqrt{\frac{M_{Z}^{2}}{M_{Z}^{2}-M_{W}^{2}}}, \\
& g^{\prime}=e \sqrt{\frac{M_{Z}^{2}}{M_{W}^{2}}}, \\
& A_{f}=\sin \theta_{f} \cos \theta_{f} \frac{m_{\tilde{f}_{2}}^{2}-m_{\tilde{f}_{1}}^{2}}{m_{f}}-\left\{\begin{array}{ll}
\mu \cot \beta, & f=u, c, t \\
\mu \tan \beta, & f=d, s, b, e, \mu, \tau
\end{array},\right. \\
& \tilde{m}_{\tilde{f}_{L}}^{2}=\cos ^{2} \theta_{f} m_{\tilde{f}_{1}}^{2}+\sin ^{2} \theta_{f} m_{\tilde{f}_{2}}^{2}-m_{f}^{2}-M_{Z}^{2} \cos 2 \beta\left(T_{3 f}-Q_{f} s_{W}^{2}\right), \\
& f=\nu_{e}, \nu_{\mu}, \nu_{\tau}, e, \mu, \tau, u, d, c, s, t, b,
\end{aligned}
$$

$\overline{2}$ In ref.[7] the value of $\mu$ is restricted in the range $0 \leq \mu$, while $-\infty<\tan \beta<+\infty$ is allowed. Due to the invariance of the lagrangian with respect to an interchange $(\mu, \tan \beta) \leftrightarrow(-\mu,-\tan \beta)$, the physics with $\mu=\mu_{0}>0$ and $\tan \beta=-\tan \beta_{0}<0$ in ref.[7] corresponds to the one with $\mu=-\mu_{0}<0$ and $\tan \beta=\tan \beta_{0}>0$ in GRACE/SUSY. 


$$
\begin{gathered}
\tilde{m}_{\tilde{f}_{R}}^{2}=\sin ^{2} \theta_{f} m_{\tilde{f}_{1}}^{2}+\cos ^{2} \theta_{f} m_{\tilde{f}_{2}}^{2}-m_{f}^{2}-M_{Z}^{2} \cos 2 \beta Q_{f} s_{W}^{2}, \\
f=e, \mu, \tau, u, d, c, s, t, b .
\end{gathered}
$$

In the last two equations of (2.15) the identities $\tilde{m}_{\tilde{e}_{L}}^{2}=\tilde{m}_{\tilde{\nu}_{e}}^{2}, \tilde{m}_{\tilde{u}_{L}}^{2}=\tilde{m}_{\tilde{d}_{L}}^{2}$, etc. are guaranteed by the relations (2.11) and (2.12). The vacuum expectation values $v_{1}$ and $v_{2}$ are given by

$$
\begin{aligned}
& v_{1}=2 \frac{M_{W}}{M_{Z}} \sqrt{M_{Z}^{2}-M_{W}^{2}} \frac{1}{e \sqrt{1+\tan ^{2} \beta}}, \\
& v_{2}=2 \frac{M_{W}}{M_{Z}} \sqrt{M_{Z}^{2}-M_{W}^{2}} \frac{\tan \beta}{e \sqrt{1+\tan ^{2} \beta}} .
\end{aligned}
$$

Note that the masses of Higgs particles, neutralinos and charginos are determined by the mass diagonalization matrices introduced in (2.4), (2.6) and (2.7), respectively. In particular, the Higgs masses are given at tree level by

$$
\begin{aligned}
M_{H^{0}, h^{0}}^{2} & =\frac{1}{2}\left[M_{A^{0}}^{2}+M_{Z}^{2} \pm \sqrt{\left(M_{A^{0}}^{2}+M_{Z}^{2}\right)^{2}-4 M_{A^{0}}^{2} M_{Z}^{2} \cos ^{2} 2 \beta}\right] \\
M_{H^{ \pm}}^{2} & =M_{A^{0}}^{2}+M_{W}^{2} .
\end{aligned}
$$

According to (2.17), the MSSM at Born order predicts the existence of a CPeven Higgs particle which is lighter than $Z$. This possibility having already been experimentally excluded, we have to make a minimal modification of the Higgs potential so that $M_{H^{0}}, M_{h^{0}}$ etc. can be treated as free parameters. For this purpose, inspired by the two-Higgs-doublet model [10,11], we introduce the effective Higgs potential (see also $[12,13]$ ) in the following way:

$$
\begin{aligned}
V^{\mathrm{eff}}= & C_{1} \mathbf{H}_{1}^{*} \mathbf{H}_{1}+C_{2} \mathbf{H}_{2}^{*} \mathbf{H}_{2}+2 C_{3} \operatorname{Re}\left(\mathbf{H}_{1} \mathbf{H}_{2}\right)+2 C_{4}\left[\operatorname{Im}\left(\mathbf{H}_{1} \mathbf{H}_{2}\right)\right]^{2} \\
& +C_{5}\left(\mathbf{H}_{1}^{*} \mathbf{H}_{1}-\mathbf{H}_{2}^{*} \mathbf{H}_{2}\right)^{2}+C_{6}\left|\mathbf{H}_{1}^{*} \mathbf{H}_{2}\right|^{2}+C_{7}\left(\mathbf{H}_{1}^{*} \mathbf{H}_{1}\right)^{2}+C_{8}\left(\mathbf{H}_{2}^{*} \mathbf{H}_{2}\right)^{2} \\
& +4 C_{9}\left(\mathbf{H}_{1}^{*} \mathbf{H}_{1}\right) \operatorname{Re}\left(\mathbf{H}_{1} \mathbf{H}_{2}\right)+4 C_{10}\left(\mathbf{H}_{2}^{*} \mathbf{H}_{2}\right) \operatorname{Re}\left(\mathbf{H}_{1} \mathbf{H}_{2}\right) \\
& +C_{11}
\end{aligned}
$$

When the coefficients $C_{i}$ take special values

$$
\begin{aligned}
& C_{1}=\tilde{m}_{1}^{2}+\mu^{2}=M_{A^{0}}^{2} \sin ^{2} \beta-\frac{M_{Z}^{2}}{2} \cos 2 \beta, \\
& C_{2}=\tilde{m}_{2}^{2}+\mu^{2}=M_{A^{0}}^{2} \cos ^{2} \beta+\frac{M_{Z}^{2}}{2} \cos 2 \beta, \\
& C_{3}=\tilde{m}_{12}^{2}=-\sin \beta \cos \beta M_{A^{0}}^{2}, \\
& C_{5}=\frac{1}{8}\left(g^{2}+g^{\prime 2}\right),
\end{aligned}
$$




$$
\begin{aligned}
& C_{6}=\frac{g^{2}}{2}, \\
& C_{4}=C_{7}=C_{8}=C_{9}=C_{10}=C_{11}=0,
\end{aligned}
$$

the above effective Higgs potential reduces to the standard one of the MSSM. Since $C_{11}$ does not contribute to the Higgs masses nor to the interactions, the effective Higgs potential depends on ten parameters $C_{i},(i=1 \sim 10)$, allowing more freedom than in the case of the original MSSM Higgs potential which contains three parameters, $\tilde{m}_{1}^{2}, \tilde{m}_{2}^{2}$ and $\tilde{m}_{12}^{2}$. The conditions at the potential minimum relate $C_{i}$ to $v_{j},(j=1,2)$. Therefore, the effective Higgs potential contains eight free input parameters ${ }^{3}$, for which we take

$$
M_{A^{0}}, \quad M_{H^{0}}, \quad M_{h^{0}}, \quad M_{H^{ \pm}}, \quad \tan \alpha, \quad C_{4}, \quad C_{9}, \quad C_{10}
$$

with the convention adopted in GRACE/SUSY

$$
-\frac{\pi}{2} \leq \alpha \leq 0
$$

In terms of our input parameters, the coefficients $C_{i}$ can be expressed as

$$
\begin{aligned}
& C_{1}=-\frac{1}{4}\left[M_{H^{0}}^{2}+M_{h^{0}}^{2}+D \cos 2 \alpha\right]-\frac{v_{2}}{4 v_{1}} D \sin 2 \alpha-\frac{v_{2}}{v_{1}} C_{3}, \\
& C_{2}=-\frac{1}{4}\left[M_{H^{0}}^{2}+M_{h^{0}}^{2}-D \cos 2 \alpha\right]-\frac{v_{1}}{4 v_{2}} D \sin 2 \alpha-\frac{v_{1}}{v_{2}} C_{3}, \\
& C_{3}=-\sin \beta \cos \beta M_{A^{0}}^{2}+v_{1} v_{2} C_{4}-v_{1}^{2} C_{9}-v_{2}^{2} C_{10}, \\
& C_{5}=-\frac{D \sin 2 \alpha}{4 v_{1} v_{2}}-\frac{3}{2} \frac{M_{A^{0}}^{2}}{v_{1}^{2}+v_{2}^{2}}-\frac{C_{3}}{v_{1} v_{2}}+\frac{3}{2} C_{4}, \\
& C_{6}=2\left(\frac{M_{H^{ \pm}}^{2}-M_{A^{0}}^{2}}{v_{1}^{2}+v_{2}^{2}}+C_{4}\right), \\
& C_{7}=-\frac{1}{v_{1}^{2}}\left[C_{1}+\frac{v_{2}}{v_{1}} C_{3}+\left(v_{1}^{2}-v_{2}^{2}\right) C_{5}+3 v_{1} v_{2} C_{9}+\frac{v_{2}^{3}}{v_{1}} C_{10}\right], \\
& C_{8}=-\frac{1}{v_{2}^{2}}\left[C_{2}+\frac{v_{1}}{v_{2}} C_{3}+\left(v_{2}^{2}-v_{1}^{2}\right) C_{5}+\frac{v_{1}^{3}}{v_{2}} C_{9}+3 v_{1} v_{2} C_{10}\right],
\end{aligned}
$$

where the vacuum expectation values $v_{i}$ are given by (2.16) 4 and

$$
D=M_{H^{0}}^{2}-M_{h^{0}}^{2}
$$

3 Compared with the MSSM, the effective Higgs potential (2.18) contains seven more parameters and consequently we need seven more new inputs.

4 (2.32) and (2.33) are the conditions for the vanishing of the linear terms in the effective Higgs potential, thus assuring that $v_{1}$ and $v_{2}$ correspond to the minimum of the potential (2.18). 
Table 1

Input parameters used in GRACE/SUSY.

\begin{tabular}{|l|l|}
\hline coupling constants & $\alpha_{e}, \alpha_{s}$ \\
\hline Gauge boson masses & $M_{Z}, M_{W}$ \\
\hline Higgs sector & $M_{H^{0}}, M_{h^{0}}, M_{A^{0}}, M_{H^{ \pm}}$ \\
& $\tan \alpha$ \\
& $C_{4}, C_{9}, C_{10}$ \\
\hline SUSY parameters & $\mu, \tan \beta, M_{1}, M_{2}, M_{3}$ \\
\hline fermion masses & $m_{u}, m_{c}, m_{t}$ \\
& $m_{d}, m_{s}, m_{b}$ \\
& $m_{e}, m_{\mu}, m_{\tau}$ \\
\hline sfermion masses & $m_{\tilde{u}_{1}}, m_{\tilde{u}_{2}}, m_{\tilde{c}_{1}}, m_{\tilde{c}_{2}}, m_{\tilde{t}_{1}}, m_{\tilde{t}_{2}}$ \\
& $m_{\tilde{d}_{1}}, m_{\tilde{s}_{1}}, m_{\tilde{b}_{1}}$ \\
& $m_{\tilde{e}_{1}}, m_{\tilde{e}_{2}}, m_{\tilde{\mu}_{1}}, m_{\tilde{\mu}_{2}}, m_{\tilde{\tau}_{1}}, m_{\tilde{\tau}_{2}}$ \\
\hline sfermion mixing angles & $\theta_{u}, \theta_{c}, \theta_{t}$, \\
& $\theta_{d}, \theta_{s}, \theta_{b}$, \\
& $\theta_{e}, \theta_{\mu}, \theta_{\tau}$ \\
\hline particle widths & $\Gamma_{W}, \Gamma_{Z}, \ldots$ \\
\hline
\end{tabular}

Combining (2.8) and (2.25), we use the following quantities as the inputs of GRACE/SUSY in the gauge and Higgs sectors,

$$
\begin{aligned}
& \alpha_{e}, \alpha_{s}, M_{W}, M_{Z}, \tan \alpha, \tan \beta, M_{A^{0}}, M_{H^{0}}, M_{h^{0}}, M_{H^{ \pm}}, \mu, M_{1}, M_{2}, M_{3}, \\
& C_{4}, C_{9}, C_{10}
\end{aligned}
$$

In addition to these quantities, all the widths of the unstable particles must also be specified as input parameters. The set of the basic input parameters used in the program system are summarized in Table 1. The magnitudes of these inputs can be changed freely by the user. A list of the names of the variables created by the system in FORTRAN codes are given in Appendix B.

A possible minimal extension of the MSSM is obtained by setting

$$
C_{4}=C_{9}=C_{10}=0
$$

and using for $\tan \alpha$ and $M_{H^{ \pm}}^{2}$ the same form as given by the tree MSSM, 


$$
\begin{aligned}
\tan 2 \alpha & =\tan 2 \beta \frac{M_{A^{0}}^{2}+M_{Z}^{2}}{M_{A^{0}}^{2}-M_{Z}^{2}}, \\
M_{H^{ \pm}}^{2} & =M_{W}^{2}+M_{A^{0}}^{2},
\end{aligned}
$$

respectively. Concerning $M_{H^{0}}$ and $M_{h^{0}}$, one can use any desired value, such as those evaluated by the one-loop MSSM.

In GRACE/SUSY, two modes are prepared for the choice of Higgs parameters. In mode 1 the strict MSSM Higgs potential is used, while in mode 2 the extended Higgs potential, (2.18), is used with the input parameters given in (2.25). The mode and the input parameters of the Higgs potential must be specified in subroutine sethptal. Note that in editing the subroutine, one can also use a different set of input parameters for the Higgs potential. See also section 4.4.2.

\subsection{Treatment of the fermion number clashing vertices}

In models of SUSY the fermion number may not be conserved at the interaction vertices when they couple to neutralinos and charginos. Note that since the fermion number of chargino is not determined by their interactions, we define the positively charged charginos as Dirac fermions.

In GRACE/SUSY we manipulate the fermion number clashing interactions by introducing the concept of G-line (GRACE-line), F-direction (fermion-direction) and M-direction (momentum-direction). The detail of the treatment for the tree case being given in ref.[14], we present here only a brief sketch of the procedure.

Since the flow of a spin one-half particle is not disconnected nor has any branch, all the external spinors are connected pairwise. When such a spinor line connecting two external spinors is stretched in a straight line and displayed horizontally, we call it a G-line. According to the G-line rule to be discussed below, we compute the G-line amplitude corresponding to each G-line. The Feynman amplitude for each graph is then obtained by multiplying all the so-constructed G-line amplitudes, the bosonic parts which connect vertices in different $\mathrm{G}$-lines and the relative phase of the graph at issue.

\section{$\underline{\text { G-line rule }}$}

Let us assume that the process contains $n_{i}$ incoming spinors and $n_{f}$ outgoing spinors. We assign the particle number 1 to $n_{i}$ to the $n_{i}$ incoming spinor particles, and $n_{i}+1$ to $n_{i}+n_{f}$ to the $n_{f}$ outgoing spinor particles. For a given fermion line connecting two external spinor particles with particle number $m$ and $n(m>n)$, we define a G-line in such a way that the particle $m$ comes from the left and the particle $n$ comes from the right endpoint. This defines 
the G-line uniquely for each fermion line belonging to a Feynman graph and there are $\left(n_{i}+n_{f}\right) / 2$ such G-lines at tree level.

On each segment of the G-line separated by the interaction vertices, we assign two directions, an M-direction and an F-direction [15]. For the notational convenience, we also introduce the notion of an A-direction, which is the direction in which spinors are ordered in the Feynman amplitude when a fermion comes in and goes out after some interactions, namely, the direction indicated by the left-oriented arrow.

\section{M-direction}

An M-direction is assigned in a natural way. For external incoming particles, their M-direction is the direction towards the vertex, while for the external outgoing particles it is the direction from the vertex. For the internal fermions, the M-direction is always taken in the A-direction (irrespective of whether the internal propagator is that of fermion or anti-fermion when seen in the Adirection).

\section{F-direction}

For Dirac particles we assign the F-direction in a natural way; for an external fermion the F-direction is the same as its M-direction, while for an external anti-fermion it is opposite to its M-direction. For the internal Dirac particle it is taken to be equal to the A-direction for fermions (when seen in the A-direction) and to the opposite direction for anti-fermions. Concerning Majorana particles which are self-charge-conjugate, how to assign their F-direction is a matter of convention. In our convention, we define the F-direction of Majorana particles by their M-direction. This means that the F-direction of the internal Majorana particle coincides with its A-direction and only the following type of the Majorana propagator appears in the G-line amplitude;

$$
<\Psi \bar{\Psi}>=\bullet \leftarrow \leftarrow \bullet=\frac{i}{p-m}
$$

where the momentum $p$ runs from right to left. Clashing of the F-direction occurs only at vertices, and the charge conjugation matrix appears only at the fermion number clashing vertices.

The above assignments of the F-direction and the M-direction uniquely identify the external spinors as shown in Table 2.

The general rule of flipping one of the F-direction at the vertex is summarized as follows

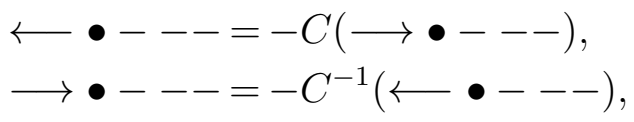


Table 2

Spinor assignment at the left and right endpoint of the G-lines.

\begin{tabular}{|l|l|l|l|l|}
\hline $\begin{array}{l}\text { F-direction } \\
\text { M-direction }\end{array}$ & $\longleftarrow \bullet$ & $\longrightarrow \bullet$ & $\bullet \longleftarrow$ & $\bullet \longrightarrow$ \\
\hline$\longleftarrow$ & $\bar{u}(p)$ & $v(p)^{T}$ & $u(p)$ & $\bar{v}(p)^{T}$ \\
$\longrightarrow$ & $\bar{v}(p)$ & $u(p)^{T}$ & $v(p)$ & $\bar{u}(p)^{T}$ \\
$---\bullet \longleftarrow$ & $=(---\bullet \longrightarrow) C^{-1}$, \\
$---\bullet \longrightarrow$ & $=(---\bullet \longleftarrow) C$,
\end{tabular}

where --- is a fermion line whose F-direction can be arbitrary. The charge conjugation matrix $C$ satisfies the following properties

$$
C^{\dagger}=C^{-1}, \quad C=-C^{T}, \quad C\left[1, \gamma_{5}, \gamma_{\mu}, \gamma_{\mu} \gamma_{5}\right]^{T} C^{-1}=\left[1, \gamma_{5},-\gamma_{\mu}, \gamma_{\mu} \gamma_{5}\right] .
$$

It is also important to note that we can choose the relative phase of the spinor wave functions such that

$$
C \bar{v}(k, \lambda)^{T}=u(k, \lambda), \quad C \bar{u}(k, \lambda)^{T}=v(k, \lambda) .
$$

The rule of reversing the particle order at a vertex is given as follows;

$$
\begin{aligned}
& B \longrightarrow \bullet \longrightarrow A=-(A \longleftarrow \bullet \longleftarrow B)^{T} \\
& B \longrightarrow \bullet \longleftarrow A=-(A \longrightarrow \bullet \longleftarrow B)^{T}, \\
& B \longleftarrow \bullet \longrightarrow A=-(A \longleftarrow \bullet \longrightarrow B)^{T} .
\end{aligned}
$$

Applying the above rules to the lagrangian of fermion number conserving Dirac fermions interactions (including chargino),

$$
\mathcal{L}_{D D}=\bar{\Psi}\left(D_{\alpha}\right) \Gamma_{D_{\alpha}, D_{\beta}} \Psi\left(D_{\beta}\right)+\text { h.c. },
$$

one obtains the G-line rule for the Dirac particles (neglecting the overall factor i)

$$
\begin{array}{ll}
D_{\alpha} \longleftarrow \bullet \longleftarrow D_{\beta} & \Gamma_{D_{\alpha}, D_{\beta}}, \\
D_{\beta} \longrightarrow \bullet \longrightarrow D_{\alpha} & -\Gamma_{D_{\alpha}, D_{\beta}}^{T} .
\end{array}
$$

Concerning the vertices including Majorana particles, corresponding to the lagrangian 


$$
\begin{aligned}
\mathcal{L}_{M D} & =\bar{\Psi}\left(M_{\alpha}\right) \Gamma_{M_{\alpha}, D_{\beta}} \Psi\left(D_{\beta}\right)+\bar{\Psi}\left(D_{\beta}\right) \Gamma_{D_{\beta}, M_{\alpha}} \Psi\left(M_{\alpha}\right) \\
\mathcal{L}_{M M} & =\sum_{\alpha \geq \beta} \bar{\Psi}\left(M_{\alpha}\right) \Gamma_{M_{\alpha}, M_{\beta}} \Psi\left(M_{\beta}\right)
\end{aligned}
$$

where $\Gamma_{D_{\alpha}, M_{\beta}} \equiv \gamma_{0} \Gamma_{M_{\beta}, D_{\alpha}}^{\dagger} \gamma_{0}$ and $D$ stands for a Dirac particle which can be a chargino, we obtain the following G-line rule,

$$
\begin{array}{ll}
M_{\alpha} \longleftarrow \bullet \longleftarrow D_{\beta} & \Gamma_{M_{\alpha}, D_{\beta}}, \\
D_{\alpha} \longleftarrow \bullet \longleftarrow M_{\beta} & \Gamma_{D_{\alpha}, M_{\beta}}, \\
M_{\alpha} \longleftarrow \bullet \longleftarrow M_{\beta} & \Gamma_{M_{\alpha}, M_{\beta}}, \quad(\alpha \geq \beta) \\
M_{\beta} \longleftarrow \bullet \longrightarrow D_{\alpha} & C \Gamma_{D_{\alpha}, M_{\beta}}^{T}, \\
D_{\beta} \longrightarrow \bullet \longleftarrow M_{\alpha} & -\Gamma_{D_{\beta}, M_{\alpha}}^{T} C^{-1} .
\end{array}
$$

Because the F-direction of Majorana particles is fixed to be equal to their Adirection, other vertices with Majorana particle having F-directions opposite to the A-direction do not appear in the G-lines. We also need the G-line rule for the fermion number violating chargino interactions with a conventional Dirac particle, whose lagrangian is given by

$$
\mathcal{L}_{C D}=\bar{\Psi}^{c}(C) \Gamma_{C, D} \Psi(D)+\bar{\Psi}(D) \Gamma_{D, C} \Psi^{c}(C)
$$

where $\Gamma_{D, C}=\gamma_{0} \Gamma_{C, D}^{\dagger} \gamma_{0}$. The rules are

$$
\begin{array}{ll}
C \longrightarrow \bullet \longleftarrow D & -C^{-1} \Gamma_{C, D}, \\
D \longleftarrow \bullet \longrightarrow C & \Gamma_{D, C} C .
\end{array}
$$

Table 2 and eq.(2.42)-(2.54) are what we need to compute the G-line amplitudes.

As Denner et al. [16] have done, it is possible to formulate the fermion number clashing G-line amplitude without using the charge conjugation matrix $C$ if one observes that the propagator with its F-direction opposite to the Adirection is given by

$$
\begin{aligned}
\bullet & \rightarrow \rightarrow \bullet=-S^{T}(-k)=-C^{-1} S(k) C, \\
& \leftarrow k
\end{aligned}
$$

and that the right-hand side of (2.50) and (2.51) is expressed in terms of the charge conjugated vertex function

$$
\Gamma_{A, B}^{c} \equiv C \Gamma_{B, A} C^{-1},
$$




$$
\begin{array}{ll}
M_{\beta} \longleftarrow \bullet \longrightarrow D_{\alpha} & \Gamma_{M_{\beta}, D_{\alpha}}^{c} C, \\
D_{\beta} \longrightarrow \bullet \longleftarrow M_{\alpha} & -C^{-1} \Gamma_{D_{\beta}, M_{\alpha}}^{c},
\end{array}
$$

In ref.[14] the equivalence of these two approaches has been demonstrated.

\section{Comments}

Several comments are in order.

We have checked and debugged the possible coding errors in GRACE/SUSY by several methods. First, the interaction vertices coded in the system, which are based on the expression presented in [8], are compared with the result computed by the algebraic manipulation program REDUCE. As another check, we have used the gauge invariance of the processes which include photon, $W^{ \pm}$, $Z$ and gluon. In this check one has to make the widths of the heavy gauge bosons vanish. By changing the value of gauge parameters, we have confirmed at one phase space point that the amplitudes of all the possible 582,102 processes with up to 6 external legs remain unchanged within the error of 1 in $10^{15}$ or less in double-precision, and 1 in $10^{30}$ or less in quadruple-precision calculation. Also further checks have been made by Boudjema and Bélanger of LAPTH [17], who compared the GRACE/SUSY results of several SUSY processes with those of CompHEP [18], the automatic computation program for the high energy processes developed by a group of Moscow State University.

Using GRACE/SUSY we have computed a number of SUSY processes and some of the results have been published. As this system allows us to deal with any SUSY particle production process at various types of high energy colliders,

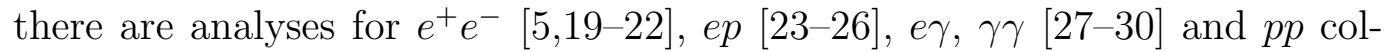
liders [31]. Note, moreover, that some works have been done with a version of GRACE/SUSY extended to the $R$-parity violating SUSY models [24-26,28$30,32]$.

GRACE/SUSY can treat as many final-state particles as possible, provided that one prepares by oneself the kinematics routine necessary for the phase space integration and the computer has an enough capacity to store the gigantic output files. The system is, however, equipped with its own kinematics library which has all the kinematics routines necessary for the analysis of up to 4-body final-state (see Appendix C).

Users can modify the program for their own interest but at their own risk. In particular, if one would like to use the other set of input parameters for SUSY 
lagrangian, for example, the trilinear coupling constants $A_{f}$ in place of $\theta_{f}$, one should prepare a program in which the parameter values are converted from the user's set to that of GRACE/SUSY given in Table 1, and call the program before the amplitude calculation starts. Note that in the system it is $m_{f} A_{f}$ but not $A_{f}$ itself that has the variable name (see Appendix B). Note also that in the sfermion sector, the unmixed physical state is obtained by setting either $\theta_{f}=0$ or $\theta_{f}=\pi / 2$. In the former case, however, the identification corresponds to $\tilde{f}_{1}=\tilde{f}_{L}$ and $\tilde{f}_{2}=\tilde{f}_{R}$, while in the latter $\tilde{f}_{1}=\tilde{f}_{R}$ and $\tilde{f}_{2}=\tilde{f}_{L}$. Since $m_{\tilde{f}_{1}} \leq m_{\tilde{f}_{2}}$ is assumed in the system, we have to set $\theta_{f}=0\left(\theta_{f}=\pi / 2\right)$ when one considers the case in which there is no mixing in the sfermion sector and the left-handed sfermion is lighter (heavier) than the right-handed one.

The program GRACE/SUSY is designed strictly within the framework of the tree level. To be more realistic, however, one can arrange the value of the input parameters so that they are subject to the renormalization group equations (RGE). A program MUSE developed by Lafage [20,33] can compute the SUSY particle masses as solutions of the coupled RGE. One can combine such a program with GRACE/SUSY to invoke improved values for the input parameters. See also ref.[34].

It is also possible to extend GRACE/SUSY so as to include new particles or new interactions such as the $R$-parity violating interaction (see, for example, [26]).

The algorithm of G-line works without any change for loop diagrams. Hence the extension of GRACE/SUSY for one-loop process is possible once one had appropriate loop evaluation routines.

In its current version of GRACE/SUSY the quark-mixing matrix is taken to be the unit matrix. Neutrinos are massless and left-handed. One may introduce the quark mixing by modifying the model files in an appropriate way.

\section{How to use GRACE/SUSY}

In this section we show how to use the program GRACE/SUSY by adopting a SUSY process $e^{+} e^{-} \rightarrow \gamma \tilde{\chi}_{1}^{+} \tilde{\chi}_{1}^{-}$as an example. This process has 28 Feynman graphs at tree level, among which 4 graphs contain even number of fermion number violating vertices.

\subsection{Definition of physical process}

First the physical process to be computed must be defined, which is given in the file "in.prc". The content of the file in.prc for the process $e^{+} e^{-} \rightarrow$ 


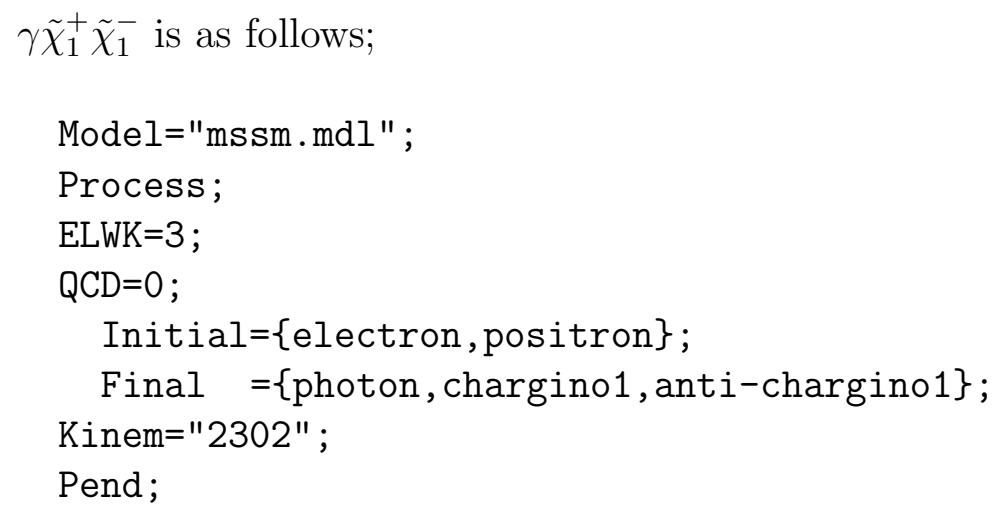

\subsubsection{Model file}

In the first line the model file is defined. Two model files are prepared, one is mssm.mdl for the minimal SUSY standard model and the other is ms.mdl for the standard model. The latter will be useful when one wants to separate the SUSY contributions from those of non-SUSY in such processes where only the SM particles appear in the external legs.

\subsubsection{Order of perturbation}

The orders of perturbation are to be defined; ELWK for the electroweak interaction and QCD for the QCD.

\subsubsection{Initial and final states}

In the lines for the initial and final states, the particle names of the process are to be given for each state according to the list given in Appendix A. One should be careful about the ordering of the particles (in particular, of massless gauge bosons) in each state. The program reads this input list and numbers all the particles from 1 to $n$ for $n$ external legs according to the order in which they appear in these two lines. These assigned numbers are in turn used to specify the integral variables in the phase space integration by the kinematic library. For example, for the 3-body final state including a single photon, the photon must appear first in the final state specification as our example shows. Otherwise the best choice of the integral variables will not be taken. See the next subsection and Appendix $\mathrm{C}$ for detail. In our example of $e^{+} e^{-} \rightarrow \gamma \tilde{\chi}_{1}^{+} \tilde{\chi}_{1}^{-}$ the input file in.prc defines the particle numbers 1 5 as $e^{-}\left(p_{1}\right), e^{+}\left(p_{2}\right), \gamma$ $\left(p_{3}\right), \chi_{1}^{+}\left(p_{4}\right)$ and $\chi_{1}^{-}\left(p_{5}\right)$. 


\subsubsection{Kinematics code number}

GRACE/SUSY has a built-in kinematics library which contains a set of kinematics routines for the phase space integration. In order to specify the kinematics routine to be used, one has to choose an appropriate code number, 2302 in our example. A list of code numbers is given in Appendix C.

\subsection{Graph generation}

The graph generation procedure starts by typing the command grc:

$$
\text { grace } \% \text { grc }
$$

All Feynman graphs for the process are generated and their information is saved in the file out.grf.

\subsection{Drawing graphs}

A Feynman graph drawer gracefig is initiated by the command:

$$
\text { grace } \% \text { gracefig }
$$

The procedure gracefig reads the graph information from the file out.grf and analyzes the structure of each graph, and then creates two windows. The output of our example process is shown in Fig.1.

\subsubsection{Drawing window of gracefig}

On the drawing window the first $m \times n$ Feynman graphs are drawn, in the unitary gauge by default, where the number of rows $m$ and the number of columns $n$ are determined automatically by the size of screen and the total number of graphs. When the size of the window is changed, the number of columns $n$ is conserved while the number of rows $m$ is adjusted so as to fit the height of the window.

\subsubsection{Control window of gracefig}

In the control window several buttons are set with which one can select through the pull-down menus several options which are listed in Table 3. 


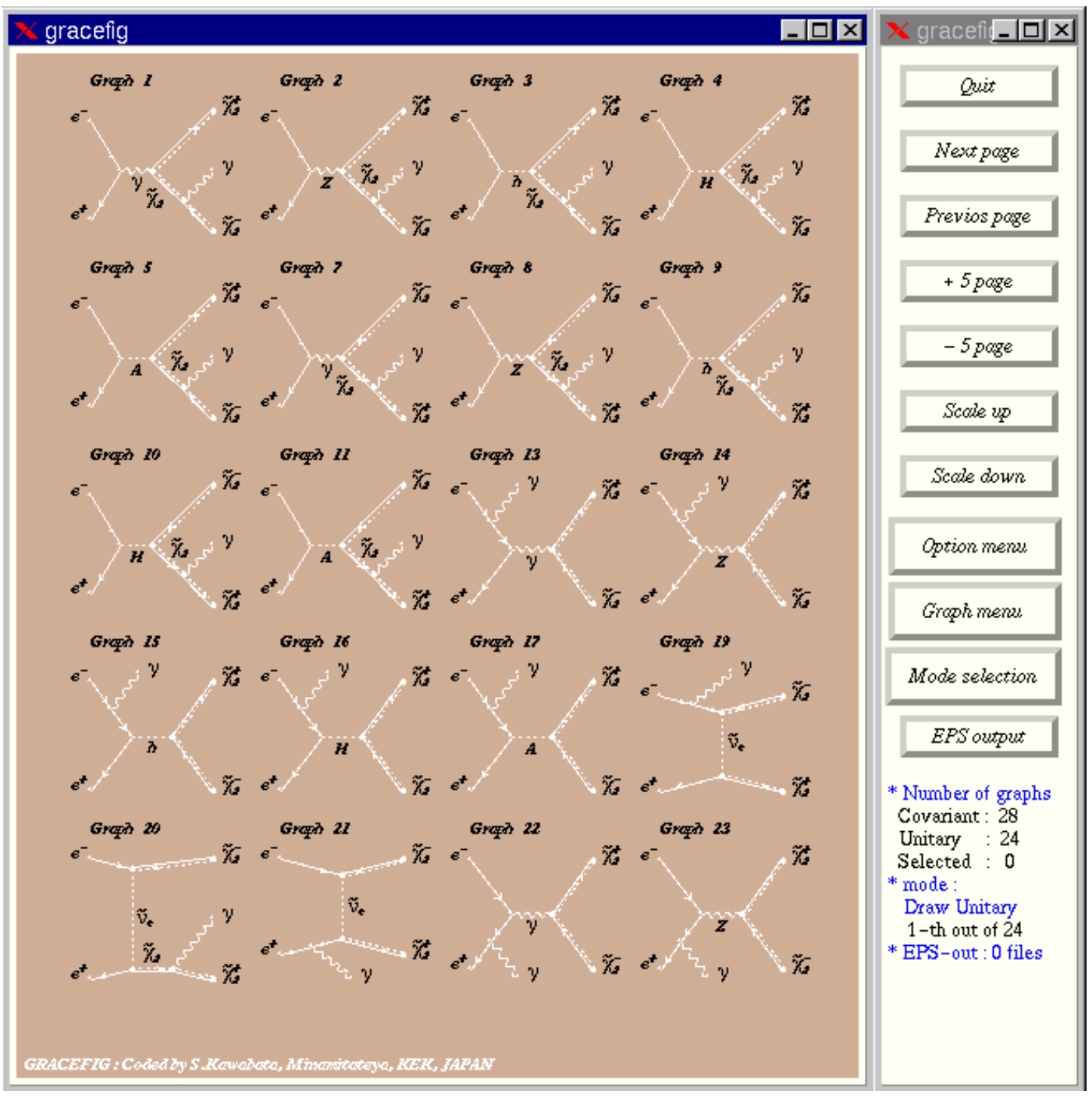

Fig. 1. Feynman graphs for $e^{+} e^{-} \rightarrow \gamma \tilde{\chi_{1}^{+}} \tilde{\chi}_{1}^{-}$in the MSSM.

Particularly one can draw all the Feynman graphs in the covariant gauge by choosing an appropriate button on the menu.

\subsubsection{To draw selected graphs}

There are two modes, "Drawing Mode" and "Select Mode". When gracefig is invoked, all Feynman graphs in the unitary gauge are displayed in the drawing mode. If one wants to see or print only some specific graphs, one can select them in the select mode by clicking their pictures in the drawing window. The background color of the selected graphs are darkened in this mode. After this selection only the selected graphs can be displayed in the drawing mode by choosing "Selected graph" in the graph menu. 
Table 3

Buttons and sub-menus of gracefig

\begin{tabular}{|c|c|}
\hline Button / Sub-menu & Function \\
\hline Quit & Exit from gracefig. \\
\hline Next page & Show next page. \\
\hline Previous page & Show previous page. \\
\hline+5 page & Show 5 -th page ahead. \\
\hline-5 page & Show 5 -th page back. \\
\hline Scale up & $\begin{array}{l}\text { Make the size of graph larger. The number of graphs } \\
\text { in a row: } n \rightarrow n-1 \text {. }\end{array}$ \\
\hline Scale down & $\begin{array}{l}\text { Make the size of graph smaller. The number of graphs } \\
\text { in a row: } n \rightarrow n+1 \text {. }\end{array}$ \\
\hline Option menu & Pull down menu. \\
\hline / Graph number & On/Off display of graph number. \\
\hline / Particle number & On/Off display of particle number. \\
\hline / Vertex number & On/Off display of vertex number. \\
\hline / Particle name & On/Off display of particle name. \\
\hline / Line number & On/Off display of internal line number. \\
\hline Graph menu & Pull down menu. \\
\hline / Covariant Gauge & Show graphs in covariant gauge. \\
\hline / Unitary Gauge & Show graphs in unitary gauge (Default). \\
\hline / Selected Graphs & Show only the selected graphs. \\
\hline Mode Selection & Pull down menu. \\
\hline / Drawing Mode & Default mode to draw the graphs. \\
\hline / Select Mode & To select a graph, click on a graph you choose. \\
\hline EPS output & $\begin{array}{l}\text { Output the current page on a file grcfig*.eps in eps } \\
\text { format. }\end{array}$ \\
\hline
\end{tabular}

\subsubsection{EPS output and insertion of a picture in a text}

When "EPS output" button is clicked, the EPS file for the current page in the drawing window is created. The name of EPS file is "grcfignn.eps", where $n n$ is the sequential number starting from 0 , and one EPS file corresponds to one page of the graphs. 


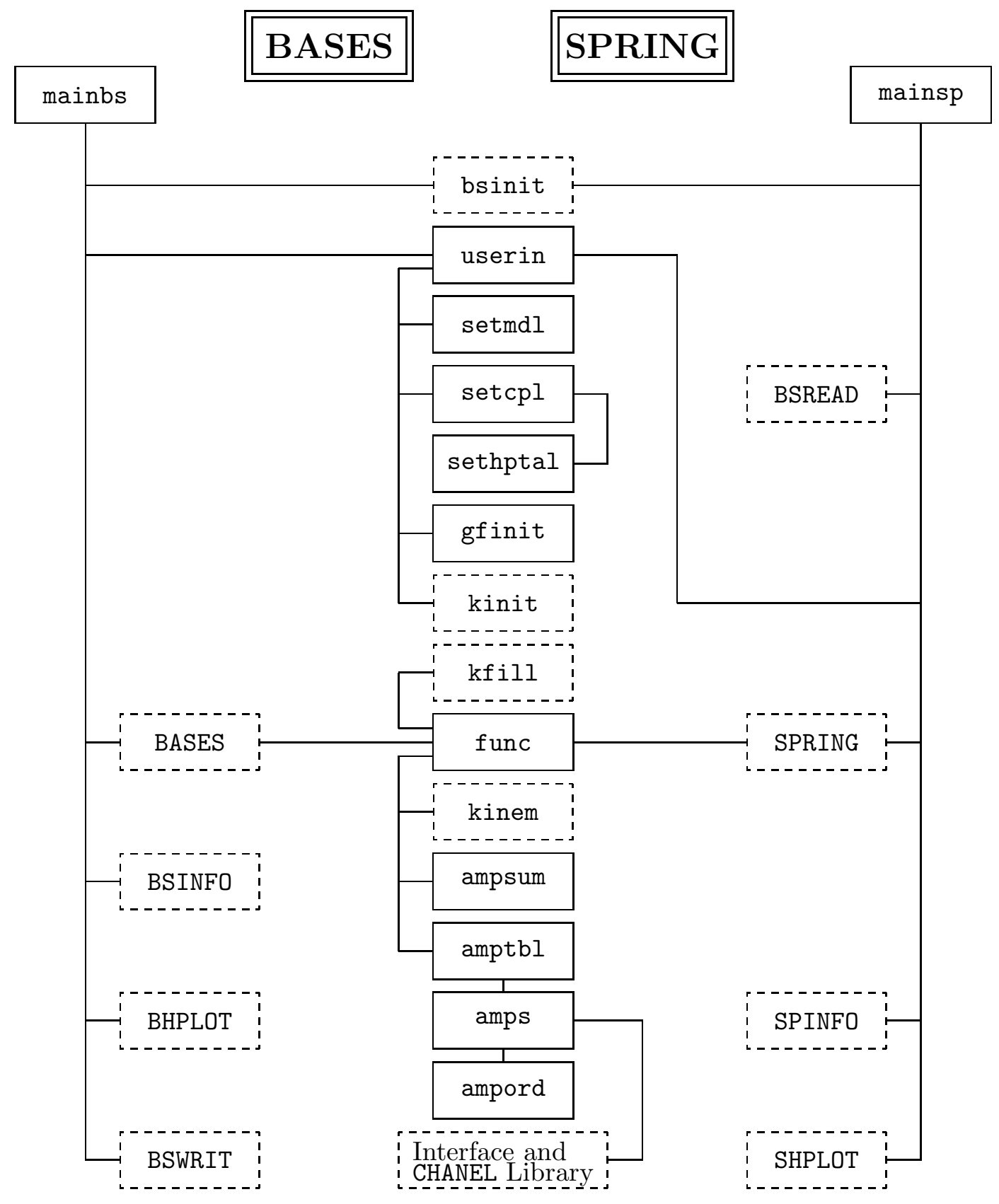

Fig. 2. Relation among the program components.

\subsection{Source code generation}

After the graph generation, by typing the command

$$
\text { grace\% grcfort }
$$

FORTRAN code and Makefile are generated, which are necessary to perform gauge independence check, the numerical integration and the event generation. 


\subsubsection{Generated source code}

Three kinds of program components are created by the system. They are used in the amplitude calculation, in the phase space integration by BASES and in the event generation by SPRING, respectively.

The interrelation among the subprograms generated by GRACE/SUSY is depicted in Fig.2, where those subprograms in the solid box are automatically generated by GRACE/SUSY, while those in the dashed box are already contained in other program packages BASES/SPRING, kinematics library, interface program library to CHANEL and program package CHANEL. This figure omits a few minor components. The components vertically located in the middle of the figure are commonly used in BASES and SPRING. The program specifications of BASES/SPRING, the interface and CHANEL are described in references $[2-4,35]$. A brief description of generated subprograms is given below:

(1) Main programs.

\begin{tabular}{lll}
\hline mainbs & (main $)$ & is the main program for the integration. \\
mainsp $\quad($ main $)$ & is the main program for the event generation. \\
gauge & $\begin{array}{l}\text { (main }) \\
\text { is the main program for the check of gauge } \\
\text { independence. }\end{array}$
\end{tabular}

(2) A set of program components for the integration by BASES and for the event generation by SPRING.

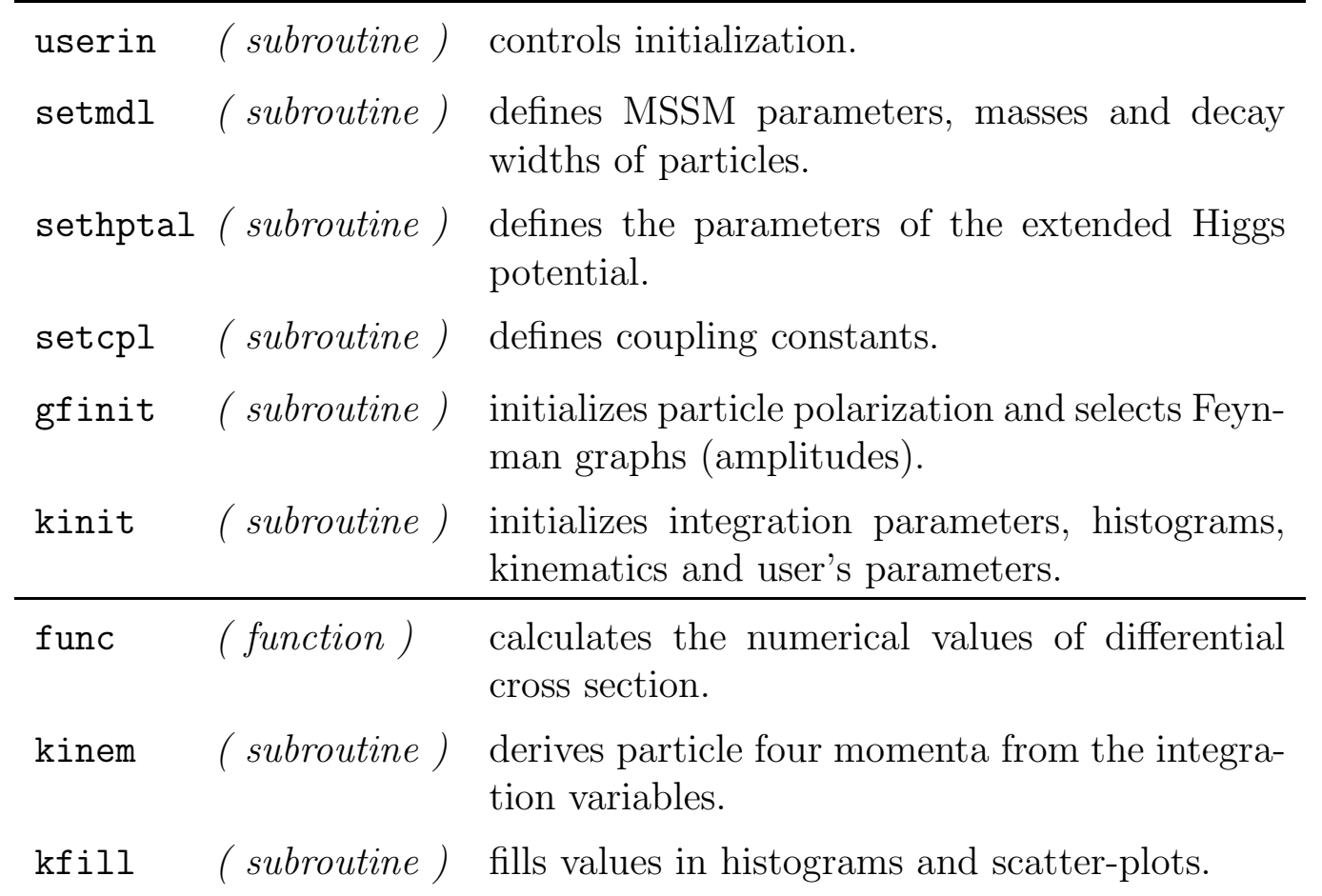




\begin{tabular}{|c|c|c|}
\hline amptbl & (subroutine) & calls amnnnn to calculate amplitudes. \\
\hline ampsum & (subroutine) & sums the square of the matrix elements \\
\hline & & over the helicity states. \\
\hline amps & ( subroutine) & calculates amplitudes. \\
\hline ampord & ( subroutine) & arranges amplitudes. \\
\hline incl1.h & (include file) & $\begin{array}{l}\text { defines the common variables for masses, am- } \\
\text { plitude tables etc. }\end{array}$ \\
\hline incl2.h & ( include file) & defines the work space for amptbl. \\
\hline inclk.h & ( include file) & transfers values of masses and a few constants. \\
\hline userout & (subroutine) & $\begin{array}{l}\text { prints the amplitude summary table. Used only } \\
\text { by gauge. }\end{array}$ \\
\hline
\end{tabular}

The subprograms userin and func are used both in the numerical integration by BASES and in the event generation by SPRING. In userin the subroutine kinit is called to initialize the kinematics routine and other parameters. The subroutines setmdl, setcpl and gfinit are also called for the initialization of physical parameters and amplitude calculation.

The function subprogram func is used for calculating the numerical value of differential cross section, where the subroutine kinem is called for calculating four-momentum vectors of external particles, and amptbl and ampsum are called for the amplitude calculation. The detailed specifications of these program components are described in refs. $[2,35]$.

\subsubsection{Setting physical parameters for numerical calculation}

Prior to running the program, one has to set the values of the input physical parameters by editing appropriate FORTRAN source codes. The parameters and corresponding subroutines to be edited are categorized as follows.

\begin{tabular}{l}
$\frac{\text { kinematical parameters }}{\text { Minit, } \text { kfill }} \begin{array}{c}\text { ketmdl, sethptal } \\
\text { sSSM parameters }\end{array}$ \\
\hline$\underline{\text { polarization and graph selection }}$ gfinit
\end{tabular}

\section{(1) Initialization routine kinit}

The values of the following parameters can be changed by editing kinit.

(a) Modification of physical parameters

Center of mass energy in $\mathrm{GeV} \cdot \sqrt{s}=1000 \mathrm{GeV}$ is a default setting, which corresponds to a description $\mathrm{w}=1000$. d0 in kinit.

Various kinematical cuts etc. 
Option flags in built-in kinematics routines ([36]).

(b) Integration parameters which control the action of BASES can be altered in kinit. The following parameters can be changed from their default values according to the required integration accuracy:

ncall The number of sampling points per iteration

acc1 The expected accuracy for the grid optimization step

itmx1 The maximum iteration number for the grid optimization

acc2 The expected accuracy for the integration step

itmx2 The maximum iteration number for the integration step

However, the following parameters are usually not to be changed:

ndim The dimensions of integral

nwild The number of wild variables

$\mathrm{xl}(i), \mathrm{xu}(i) \quad$ The lower and upper bounds of integration variable $\mathrm{x}(i)$

ig $(i) \quad$ The grid optimization flag for $i$-th variable

(c) Initialization of histograms and scatter-plots

If the user wants histograms for some physical variables, the initialization routines xhinit and dhinit should be called for each histogram and scatter-plot, respectively.

The system automatically generates source code to call these routines so as to construct histograms with respect to each integration variable, $\mathrm{x}(\mathrm{i}), \quad \mathrm{i}=1 \sim$ ndim and the energies and angles of final particles. If the user does not need these default histograms, one may delete the corresponding FORTRAN statements in kinit. When the user needs more histograms for some distributions, one should add lines to call xhinit or dhinit. These routines are to be written in the following format

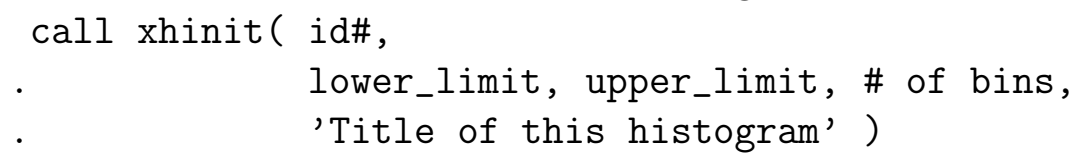

and

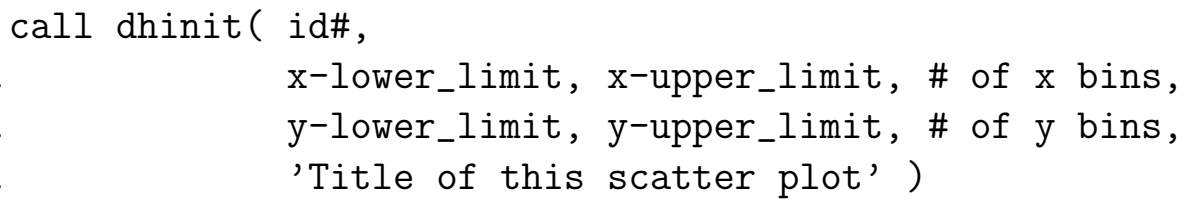

where $1 \leq \mathrm{id \#} \leq 99$.

(2) Filling histograms and scatter-plots in kfill

In the generated kfill by GRACE/SUSY, histograms for all integration variables and scatter-plots for all combinations of them are filled by calling 
xhfill and dhfill, respectively. If one changes the initialization of histograms and scatter-plots in kinit, their filling parts should be changed accordingly. These routines are to be called as

call xhfill ( id\#, v, func )

call dhfill (id\#, vx, vy, func )

(3) Subprogram setmdl

The MSSM parameters, masses and widths of particles are defined in setmdl subroutine. The variable names of all the parameters are listed in Appendix B. As an example we show how to set the MSSM parameters in the case $\left(\mu, M_{2}, \tan \beta, m_{\tilde{\nu_{e}}}\right)=(-300 \mathrm{GeV}, 200 \mathrm{GeV}, 10,150 \mathrm{GeV})$.

$\begin{array}{llr}\text { tanbe } & = & 10 . \mathrm{d} 0 \\ \mathrm{xmu} & = & -300 . \mathrm{d} 0 \\ \mathrm{xm} 2 & = & 200 . \mathrm{d} 0 \\ \operatorname{amsn}(1) & = & 150 . \mathrm{d} 0\end{array}$

(4) Subprogram sethptal

In this subprogram one has to specify the Higgs potential to be used in the calculation by assigning a value 1 or 2 to the variable ihiggs. See section 2.4 for detail.

- ihiggs = 1: mode 1; MSSM Higgs potential

- ihiggs $=2$ : mode 2 ; general extended Higgs potential (default)

The default value is ihiggs $=2$, in which case one has to give values of the additional seven input parameters, for example,

$$
\begin{array}{ll}
\operatorname{amh} 1 & =115 \cdot \mathrm{d} 0 \\
\text { amh2 } & =300 \cdot \mathrm{d} 0 \\
\text { amhc } & =350 . \mathrm{d} 0 \\
\mathrm{al} & =-0.37 \mathrm{~d} 0 \\
\mathrm{c} 4 & =0 . \mathrm{d} 0 \\
\mathrm{c} 9 & =0 . \mathrm{d} 0 \\
\mathrm{c} 10 & =0 . \mathrm{d} 0
\end{array}
$$

(5) Subprogram gfinit

(a) Selection of graphs.

The contribution from a graph is calculated when its flag is set to be jselg = 1 in gfinit. For example, if one wants to know the contributions only from the sneutrino exchange (graphs 19, 20, 21 and 28 in Fig.1), one should write

$$
\begin{aligned}
& \text { do } 10 \mathrm{n} 1=1 \text {, ngraph } \\
& \text { jselg(n1) }=0 \\
& 10 \begin{array}{c}
\text { continue } \\
j \operatorname{selg}(19)=1 \\
j \operatorname{selg}(20)=1 \\
j \operatorname{selg}(21)=1 \\
j \operatorname{selg}(28)=1
\end{array}
\end{aligned}
$$


The selection of graphs should respect gauge invariance, otherwise the cross section is physically not sensible. It should be noted that this specification of graphs is independent from the graph selection in the Feynman graph drawer gracefig.

(b) Set of polarization.

As the helicity amplitude technique is used in GRACE/SUSY, the beam polarization can be specified. The polarization sum is performed by setting the flags $j$ hs (i) and $j$ he(i) for particle $i$ having spin $s_{i}$, where $0 \leq j h s(i) \leq j h e(i) \leq 2 s_{i}$. In default the unpolarized cross section is assumed, which corresponds to setting the flags for the initial $e^{-}$(particle 1) and $e^{+}$(particle 2) as

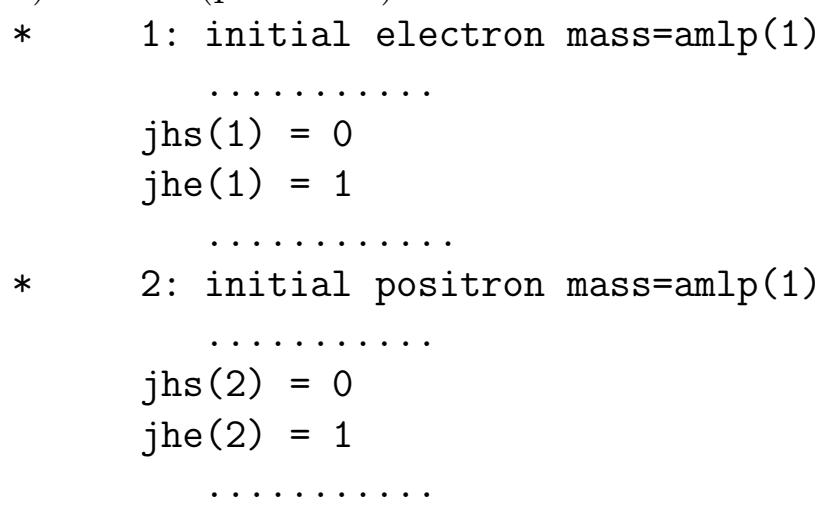

When we consider the initial $e^{-}$beam polarization, for example, they should be changed to

$$
\begin{aligned}
& \text { jhs }(1)=0 \\
& \text { jhe (1) }=0
\end{aligned}
$$

for helicity $=-1$ and

$$
\begin{aligned}
& \text { jhs }(1)=1 \\
& \text { jhe }(1)=1
\end{aligned}
$$

for helicity $=+1$.

Similarly, one can choose polarization vectors of external gauge bosons by putting $j h s(i), j h e(i)=0,1$ (transverse), 2(longitudinal: only for massive gauge bosons). In the default the linearly polarized states are realized as combinations of the transverse polarizations. When one wants to take the circular polarization for a photon, an option switch jcpol should be changed from 0 to 1.

(c) Gauge parameters.

Without a specification the unitary gauge is used in the integration over the phase space. However, one can select covariant gauge $\left(R_{\xi}\right.$-gauge) with the gauge parameters having arbitrary values.

The selection of gauge is controlled by integer variables igauwb, igauzb, igauab and igaugl which correspond to gauge bosons $W^{ \pm}, Z, \gamma$ and gluon, 
respectively. When these parameters are set to be 0 , unitary gauge is selected. Otherwise they should be igauwb $=1$, igauzb $=2$, igauab $=3$ and igaugl $=4$. The value of each gauge parameter should be given by agauge(igauxx), where $x x$ is one of $w b, z b, a b$ or gl.

In the file gfinit.f one will find the following lines which select the unitary gauge.

* Gauge parametes (default is unitary gauge)

igauab $=0$

igauwb $=0$

igauzb $=0$

igaugl $=0$

agauge $(0)=1.0 \mathrm{~d} 20$

In a general covariant gauge these lines should be changed as follows;

* Gauge parametes (covariant gauge)

igauwb $=1$

igauzb $=2$

igauab $=3$

igaugl $=4$

agauge $($ igauwb $)=\ldots$

agauge $($ igauzb $)=\ldots$

agauge $($ igauab $)=\ldots$

agauge $($ igaugl $)=\ldots$

\subsection{Compilation and gauge independence check}

As described in a previous subsection, Makefile is also generated by the command grcfort. The command make creates the executable files gauge, integ and spring.

Before calculating the cross section a preliminary test of the program source should be done. In GRACE/SUSY system gauge independence test is prepared for this purpose. The numerical values of the differential cross section at one sample point in the phase space are calculated both in the covariant and unitary gauge, and the consistency between these two values is examined. By executing gauge, the contributions to the cross section at one phase space point in unitary gauge (ans1) and in covariant gauge (ans2), and their difference (ans1/ans2-1) are shown as the following example demonstrates.

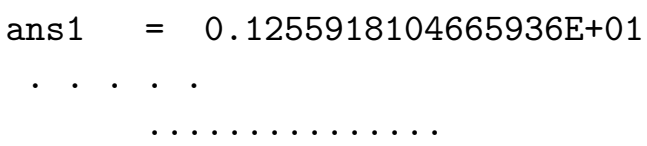




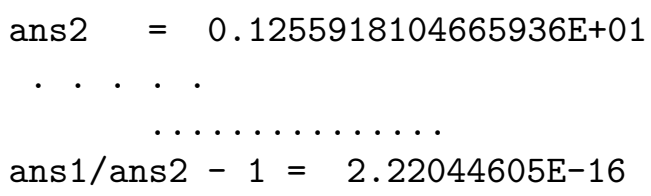

The relative difference should be around $10^{-16}$ in the double-precision computation and around $10^{-30}$ in the quadruple-precision.

It should be noted that even though this gauge independence check shows consistency between the two gauges, it does not necessarily guarantee a complete gauge independence, since it tests only at a specific point in the phase space. It is recommended to repeat a similar check at several points in phase space by changing the variable $\mathrm{x}(i)$ in gauge. $f$ from their default value of 0.45 to other value lying in the range $0<\mathrm{x}(i)<1$.

\subsection{Integration}

After a successful test of gauge independence, one can proceed to the numerical integration by BASES, whose details can be found in ref.[2]. By the make command one has already created the executable files integ for the integration and spring for event generation.

For integration the command integ is used.

$$
\text { grace } \% \text { integ }
$$

The integration parameters and the result of the integration at each iteration are shown on the screen, and at the same time they are automatically stored in the file bases.result together with the histogram outputs. If one uses a built-in kinematics routine, the cross section is given in unit of pico-barn(pb).

The numerical integration is performed by two successive steps, the first is the grid optimization step and the second the integration step. In the former the gridding of the phase space in hypercubes is optimized for the integrand, while in the latter an accurate estimate of the integral is evaluated with frozen grids determined in the first step.

Before termination of the integration procedure, BASES creates a binary file bases.data and writes the probability information on it, which is used for the event generation.

The system will issue WARNING messages when the convergence is not well established. It is always recommended to look at the integration result carefully, particularly over the convergence behavior both for the grid optimization and 
integration step. When the convergence accuracy fluctuates largely from one iteration to another, and/or, when in some cases it suddenly jumps up to a value far from the preceding iterations, the resultant estimate of integral is not reliable. There are two possible origins of such a behavior; one is due to too small number of sampling points and the other, more serious, is due to an unsuitable choice of the integration variables for the integrand. In the latter case one should use a better set of the integral variables.

\subsection{Event generation}

The event generation starts with the command

$$
\text { grace } \% \text { spring }
$$

Then SPRING reads the probability information from the binary file bases . data and asks the number of events with the following prompt:

Number of events ?

The user must type the number of events to be generated.

If the sample point does not meet the generation criteria, such a point is discarded as a "failed generation". The event generation will run until a given number of events are generated or the number of failed generations exceeds its given maximum mxtry, whose default value is 50 .

In order to estimate the computing time for the event generation, one can refer to the expected generation time given in the information of BASES output, where the generation efficiency of $70 \%$ is assumed.

\subsubsection{Analysis of events}

In the event generation four-momentum vectors of the final particles are automatically stored in $\operatorname{vec}(i, n)$ in mainsp, where $n$ stands for the particle number and $i=1 \sim 4$ correspond to the three-momentum $(1,2,3)$ and the energy (4) of the particle.

To create histograms of some variables of the generated events, one has to initialize them before the generation loop and fill them after each call of spring. This kind of histograms is called as an "additional histogram" in contrast to the "original histogram" which is filled both in the integration and event generation steps. In the original histograms one can see how the generated events reproduce those distributions produced by the integration, while in the additional histogram only the frequency distribution can be seen as in the usual 
histogram. An example for saving the four-momentum vectors and creating additional histograms is given below.

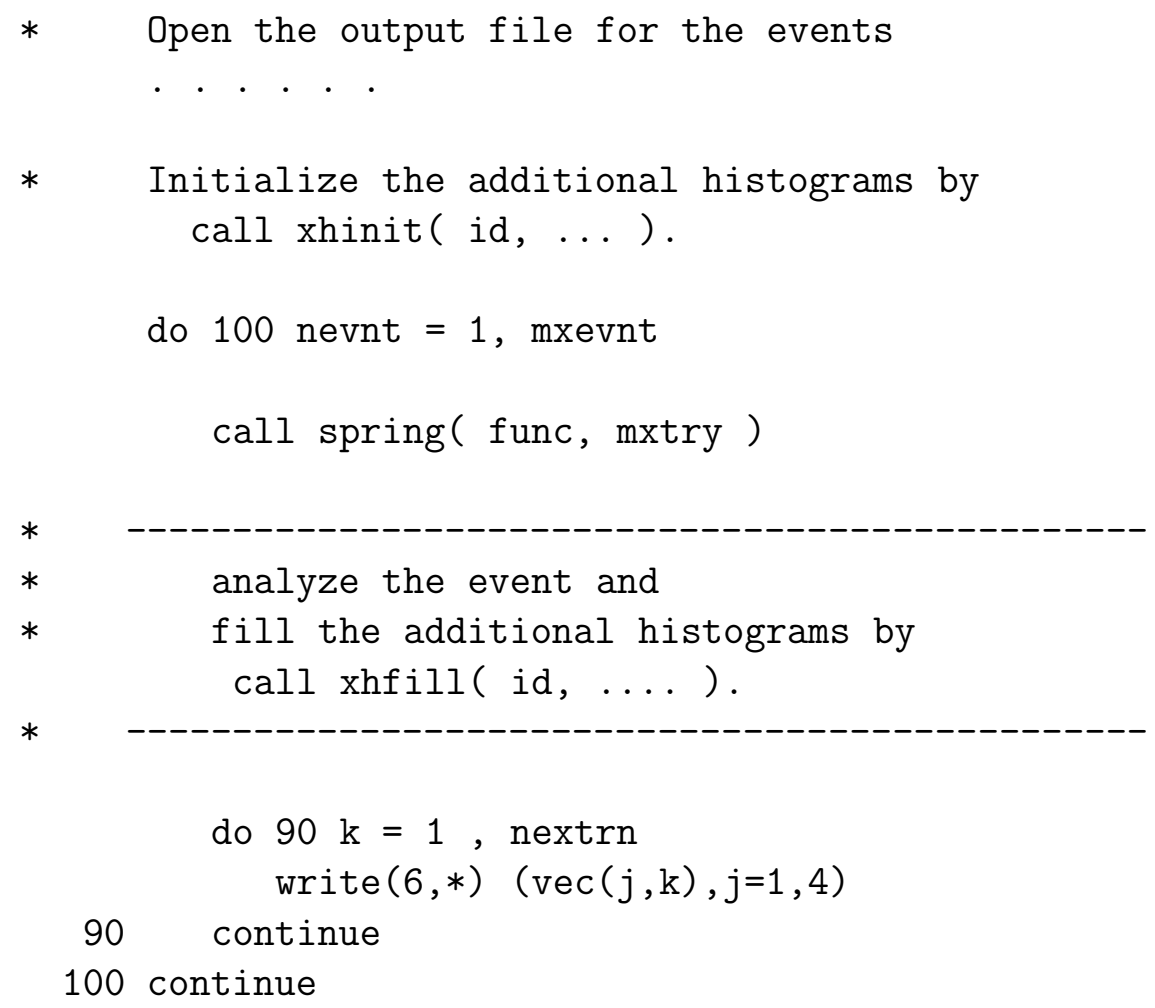

\subsubsection{Output from SPRING}

The output from SPRING is written on the file named spring.result, which consists of the general information, original and additional histograms, scatterplots, and the number-of-trials distribution. In the last distribution one can see the generation efficiency. It is recommended to check if this distribution has a sharp peak in the first bin. If this is not the case, the integration should be carried out again with more sampling points or with more iterations.

\section{Acknowledgements}

We would like to thank F. Boudjema and G. Bélanger for checking parts of the program and reading the manuscript. We would like to thank also D. PerretGallix for his interest in this work and for encouragement. This work was partly supported by Japan Society for Promotion of Science under the Grant-in-Aid for Scientific Research B ( No.11440083 ). 


\section{A List of particles in MSSM}

The particles appearing in MSSM and their variable names are listed in two tables below. These variable names are to be used in specifying the process in in. prc.

\begin{tabular}{|l|l|}
\hline particle & variable name \\
\hline$W^{+}\left(W^{-}\right)$ & W-plus (W-minus) \\
$Z$ & Z \\
gluon & gluon \\
\hline$\nu_{e}\left(\overline{\nu_{e}}\right)$ & nu-e (nu-e-bar) \\
$e^{-}\left(e^{+}\right)$ & electron (positron) \\
$\nu_{\mu}\left(\overline{\nu_{\mu}}\right)$ & nu-mu (nu-mu-bar) \\
$\mu^{-}\left(\mu^{+}\right)$ & muon (anti-muon) \\
$\nu_{\tau}\left(\overline{\nu_{\tau}}\right)$ & nu-tau (nu-tau-bar) \\
$\tau^{-}\left(\tau^{+}\right)$ & tau (anti-tau) \\
\hline$u(\bar{u})$ & u (u-bar) \\
$d(\bar{d})$ & d (d-bar) \\
$c(\bar{c})$ & c (c-bar) \\
$s(\bar{s})$ & s (s-bar) \\
$t(\bar{t})$ & t (t-bar) \\
$b(\bar{b})$ & b (b-bar) \\
\hline$h^{0}$ & Higgs1 \\
$H^{0}$ & Higgs2 \\
$A^{0}$ & Higgs3 \\
$H^{+}\left(H^{-}\right)$ & Higgs-plus (Higgs-minus) \\
\hline
\end{tabular}




\begin{tabular}{|c|c|}
\hline particle & variable name \\
\hline $\begin{array}{l}{\tilde{\chi_{1}^{+}}}^{+}\left(\tilde{\chi_{1}^{-}}\right) \\
\tilde{\chi_{2}^{+}}\left(\tilde{\chi_{2}^{-}}\right)\end{array}$ & $\begin{array}{l}\text { chargino1 (anti-chargino1) } \\
\text { chargino2 (anti-chargino2) }\end{array}$ \\
\hline $\begin{array}{l}\tilde{\chi}_{1}^{0} \\
\tilde{\chi}_{2}^{0} \\
\tilde{\chi}_{3}^{0} \\
\tilde{\chi}_{4}^{0}\end{array}$ & $\begin{array}{l}\text { neutralino1 } \\
\text { neutralino2 } \\
\text { neutralino3 } \\
\text { neutralino4 }\end{array}$ \\
\hline$\tilde{\nu_{e}}\left(\overline{\tilde{\nu}_{e}}\right)$ & snu-e (anti-snu-e) \\
\hline$\tilde{\nu_{\mu}}\left(\overline{\widetilde{\nu_{\mu}}}\right)$ & snu-mu (anti-snu-mu) \\
\hline$\tilde{\nu_{\tau}}\left(\overline{\nu_{\tau}}\right)$ & snu-tau (anti-snu-tau) \\
\hline$\tilde{e_{1}^{-}}\left(\tilde{e_{1}^{+}}\right)$ & selectron1 (anti-selectron1) \\
\hline$\tilde{e_{2}^{-}}\left(\tilde{e_{2}^{+}}\right)$ & selectron2 (anti-selectron2) \\
\hline$\tilde{\mu_{1}^{-}}\left(\tilde{\mu_{1}^{+}}\right)$ & smuon1 (anti-smuon1) \\
\hline$\tilde{\mu_{2}^{-}}\left(\tilde{\mu_{2}^{+}}\right)$ & smuon2 (anti-smuon2) \\
\hline$\tilde{\tau_{1}^{-}}\left(\tilde{\tau_{1}^{+}}\right)$ & stau1 (anti-stau1) \\
\hline$\tilde{\tau_{2}^{-}}\left(\tilde{\tau_{2}^{+}}\right)$ & stau2 (anti-stau2) \\
\hline$\tilde{u_{1}}\left(\overline{u_{1}}\right)$ & su1 (anti-su1) \\
\hline$\tilde{u_{2}}\left(\overline{\tilde{u_{2}}}\right)$ & su2 (anti-su2) \\
\hline$\tilde{d}_{1}\left(\overline{\tilde{d}_{1}}\right)$ & sd1 (anti-sd1) \\
\hline$\tilde{d}_{2}\left(\overline{\tilde{d}_{2}}\right)$ & sd2 (anti-sd2) \\
\hline$\tilde{c_{1}}\left(\overline{\tilde{c_{1}}}\right)$ & $\operatorname{sc1}(\operatorname{anti-sc1})$ \\
\hline$\tilde{c_{2}}\left(\overline{\tilde{c_{2}}}\right)$ & sc2 (anti-sc2) \\
\hline$\tilde{s_{1}}\left(\overline{\tilde{s_{1}}}\right)$ & ss1 (anti-ss1) \\
\hline$\tilde{s_{2}}\left(\overline{s_{2}}\right)$ & ss2 (anti-ss2) \\
\hline$\tilde{t_{1}}\left(\overline{t_{1}}\right)$ & st1 (anti-st1) \\
\hline$\tilde{t_{2}}\left(\overline{\widetilde{t_{2}}}\right)$ & st2 (anti-st2) \\
\hline$\tilde{b_{1}}\left(\overline{b_{1}}\right)$ & $\operatorname{sb1}($ anti-sb1) \\
\hline$\tilde{b_{2}}\left(\overline{\vec{b}_{2}}\right)$ & $\operatorname{sb2}($ anti-sb2) \\
\hline
\end{tabular}




\section{B Name List of GRACE/SUSY Parameters}

The variable names used in GRACE/SUSY are summarized. All mass variables. widths and the dimensional coupling constant $\mu$ must be given in unit of $\mathrm{GeV}$. Those variables whose magnitude can be specified by the user in setmdl.f and sethptal.f are indicated by an asterisk "*" at the end of the row. The value of other variables should not be changed by the user. Such a modification may cause internal inconsistencies.

gauge bosons

\begin{tabular}{|l|l|l|l|}
\hline category & notation & variable name in GRACE/SUSY & \\
\hline gauge boson masses & $M_{Z}, M_{W}$ & amz, amw & $*$ \\
gauge boson widths & $\Gamma_{Z}, \Gamma_{W}$ & agz, agw & $*$ \\
coupling & $\alpha_{e}, \alpha_{s}$ & alpha, alphas & $*$ \\
& $e, g_{s}$ & ge, gs & \\
mixing angle & $g, g^{\prime}$ & gg, gz & \\
\hline
\end{tabular}

Ordinary fermions

\begin{tabular}{|l|l|l|l|}
\hline fermion mass & $m_{u}, m_{c}, m_{t}$ & $\operatorname{amuq}(1), \operatorname{amuq}(2), \operatorname{amuq}(3)$ & $*$ \\
& $m_{d}, m_{s}, m_{b}$ & $\operatorname{amdq}(1), \operatorname{amdq}(2), \operatorname{amdq}(3)$ & $*$ \\
& $m_{e}, m_{\mu}, m_{\tau}$ & $\operatorname{amlp}(1), \operatorname{amlp}(2), \operatorname{amlp}(3)$ & $*$ \\
\hline fermion widths & $\Gamma_{u}, \Gamma_{c}, \Gamma_{t}$ & $\operatorname{aguq}(1), \operatorname{aguq}(2), \operatorname{aguq}(3)$ & $*$ \\
& $\Gamma_{d}, \Gamma_{s}, \Gamma_{b}$ & $\operatorname{agdq}(1), \operatorname{agdq}(2), \operatorname{agdq}(3)$ & $*$ \\
& $\Gamma_{e}, \Gamma_{\mu}, \Gamma_{\tau}$ & $\operatorname{aglp}(1), \operatorname{aglp}(2), \operatorname{aglp}(3)$ & $*$ \\
\hline
\end{tabular}

$\underline{\text { Higgs bosons }}$ 


\begin{tabular}{|l|l|l|l|}
\hline SUSY parameters & $\mu$ & $\mathrm{xmu}$ & $*$ \\
\hline Higgs masses & $\begin{array}{l}M_{A^{0}} \\
M_{h}, M_{H}, M_{H^{ \pm}}\end{array}$ & $\begin{array}{l}\text { amh3 } \\
\text { amh1, amh2, amhc }\end{array}$ & $*$ \\
\hline Higgs widths & $\Gamma_{h}, \Gamma_{H}, \Gamma_{A}, \Gamma_{H^{ \pm}}$ & agh1, agh2, agh3, aghc & $*$ \\
\hline mixing angle & $\alpha, \cos \alpha, \sin \alpha$ & al, ca, sa & $*$ \\
& $\cos \beta, \sin \beta, \tan \beta, \cot \beta$ & $\mathrm{cb}, \mathrm{sb}, \mathrm{tanbe}, \operatorname{cotbe}$ & $*$ \\
\hline
\end{tabular}

\section{$\underline{\text { Chargino }}$}

\begin{tabular}{|l|l|l|l|}
\hline SUSY parameters & $\mu, M_{2}$ & $\mathrm{xmu}, \mathrm{xm} 2$ & $*$ \\
\hline chargino masses & $m_{\tilde{\chi}_{1}^{ \pm}}, m_{\tilde{\chi}_{2}^{ \pm}}$, & $\operatorname{amsw}(1), \operatorname{amsw}(2)$ & \\
\hline chargino widths & $\Gamma_{\tilde{\chi}_{1}^{ \pm}}, \Gamma_{\tilde{\chi}_{2}^{ \pm}}$ & $\operatorname{agsw}(1), \operatorname{agsw}(2)$ & $*$ \\
\hline chargino mixing angles & $\sin \phi_{L}, \cos \phi_{L}, \epsilon_{L}$ & $\mathrm{sphl}, \mathrm{cphl}, \mathrm{eps} 1$ & \\
& $\sin \phi_{R}, \cos \phi_{R}$ & $\mathrm{sphr}, \mathrm{cphr}$ & \\
\hline
\end{tabular}

$\underline{\text { Neutralino }}$

\begin{tabular}{|l|l|l|l|}
\hline SUSY parameters & $\mu, M_{1}, M_{2}$ & $\mathrm{xmu}, \mathrm{xm} 1, \mathrm{xm} 2$ & $*$ \\
\hline neutralino masses & $m_{\tilde{\chi}_{i}^{0}}$ & $\operatorname{amnl}(i)$ & \\
\hline neutralino widths & $\Gamma_{\tilde{\chi}_{i}^{0}}$ & $\operatorname{agnl}(i)$ & $*$ \\
\hline neutralino mixing matrix & $\left(\mathcal{O}_{N}\right)_{i j}, \eta_{i}$ & $\operatorname{ogmn}(i, j), \operatorname{etan}(i)(\operatorname{complex})$ & \\
\hline
\end{tabular}

$\underline{\text { Gluino }}$

\begin{tabular}{|l|l|l|l|}
\hline gluino mass & $m_{\tilde{g}}=M_{3}$ & amgl $=\mathrm{xm3}$ & $*$ \\
gluino width & $\Gamma_{\tilde{g}}$ & aggl & $*$ \\
\hline
\end{tabular}

$\underline{\text { Sfermion }}$ 


\begin{tabular}{|c|c|c|c|}
\hline sfermion masses & $\begin{array}{l}m_{\tilde{u}_{i}}, m_{\tilde{c}_{i}}, m_{\tilde{t}_{i}} \\
m_{\tilde{d}_{i}}, m_{\tilde{s}_{i}}, m_{\tilde{b}_{i}} \\
m_{\tilde{\nu}_{e}}, m_{\tilde{\nu}_{\mu}}, m_{\tilde{\nu}_{\tau}} \\
m_{\tilde{e}_{i}}, m_{\tilde{\mu}_{i}}, m_{\tilde{\tau}_{i}}\end{array}$ & $\begin{array}{l}\operatorname{amsu}(1, i), \operatorname{amsu}(2, i), \operatorname{amsu}(3, i) \\
\operatorname{amsd}(1, i), \operatorname{amsd}(2, i), \operatorname{amsd}(3, i) \\
\operatorname{amsn}(1), \operatorname{amsn}(2), \operatorname{amsn}(3) \\
\operatorname{amsl}(1, i), \operatorname{amsl}(2, i), \operatorname{amsl}(3, i)\end{array}$ & $\begin{array}{l}* \\
* \\
* \\
*\end{array}$ \\
\hline sfermion widths & $\begin{array}{l}\Gamma_{\tilde{u}_{i}}, \Gamma_{\tilde{c}_{i}}, \Gamma_{\tilde{t}_{i}} \\
\Gamma_{\tilde{d}_{i}}, \Gamma_{\tilde{s}_{i}}, \Gamma_{\tilde{b}_{i}} \\
\Gamma_{\tilde{\nu}_{e}}, \Gamma_{\tilde{\nu}_{\mu}}, \Gamma_{\tilde{\nu}_{\tau}} \\
\Gamma_{\tilde{e}_{i}}, \Gamma_{\tilde{\mu}_{i}}, \Gamma_{\tilde{\tau}_{i}}\end{array}$ & $\begin{array}{l}\operatorname{agsu}(1, i), \operatorname{agsu}(2, i), \operatorname{agsu}(3, i) \\
\operatorname{agsd}(1, i), \operatorname{agsd}(2, i), \operatorname{agsd}(3, i) \\
\operatorname{agsn}(1), \operatorname{agsn}(2), \operatorname{agsn}(3) \\
\operatorname{agsl}(1, i), \operatorname{agsl}(2, i), \operatorname{agsl}(3, i)\end{array}$ & $\begin{array}{l}* \\
* \\
*\end{array}$ \\
\hline $\begin{array}{l}\text { SUSY-breaking } \\
\text { parameters }\end{array}$ & $\begin{array}{l}m_{u} A_{u}, m_{c} A_{c}, m_{t} A_{t} \\
m_{d} A_{d}, m_{s} A_{s}, m_{b} A_{b} \\
m_{e} A_{e}, m_{\mu} A_{\mu}, m_{\tau} A_{\tau}\end{array}$ & $\begin{array}{l}\operatorname{xauq}(1), \operatorname{xauq}(2), \operatorname{xauq}(3) \\
\operatorname{xadq}(1), \operatorname{xadq}(2), \operatorname{xadq}(3) \\
\operatorname{xalp}(1), \operatorname{xalp}(2), \operatorname{xalp}(3)\end{array}$ & \\
\hline sfermion mixing & $\begin{array}{l}\sin \theta_{\tilde{u}}, \sin \theta_{\tilde{c}}, \text { angles } \sin \theta_{\tilde{t}} \\
\cos \theta_{\tilde{u}}, \cos \theta_{\tilde{c}}, \cos \theta_{\tilde{t}} \\
\sin \theta_{\tilde{d}}, \sin \theta_{\tilde{s}}, \sin \theta_{\tilde{b}} \\
\cos \theta_{\tilde{d}}, \cos \theta_{\tilde{s}}, \cos \theta_{\tilde{b}} \\
\sin \theta_{\tilde{e}}, \sin \theta_{\tilde{\mu}}, \sin \theta_{\tilde{\tau}} \\
\cos \theta_{\tilde{e}}, \cos \theta_{\tilde{\mu}}, \cos \theta_{\tilde{\tau}}\end{array}$ & $\begin{array}{l}\operatorname{shuq}(1), \operatorname{shuq}(2), \operatorname{shuq}(3) \\
\operatorname{chuq}(1), \operatorname{chuq}(2), \operatorname{chuq}(3) \\
\operatorname{shdq}(1), \operatorname{shdq}(2), \operatorname{shdq}(3) \\
\operatorname{chdq}(1), \operatorname{chdq}(2), \operatorname{chdq}(3) \\
\operatorname{shlp}(1), \operatorname{shlp}(2), \operatorname{shlp}(3) \\
\operatorname{chlp}(1), \operatorname{chlp}(2), \operatorname{chlp}(3)\end{array}$ & $\begin{array}{l}* \\
* \\
* \\
* \\
* \\
*\end{array}$ \\
\hline
\end{tabular}

\section{Kinematics Library}

\section{C.1 Cross section formula}

The volume element for each of final particles is given by

$$
\int \frac{\mathrm{d}^{3} p}{2 E(2 \pi)^{3}}
$$

where $\mathbf{p}$ is the three momentum of the particle and $E$ is its energy.

In a collision of two particles labeled by 1 and 2, the flux is given by

$$
\text { flux }=v_{r e l} 2 E_{1} 2 E_{2}, \quad v_{r e l}=\left|\frac{\mathbf{p}_{1}}{E_{1}}-\frac{\mathbf{p}_{2}}{E_{2}}\right|,
$$


with $v_{\text {rel }}$ being the relative velocity of particles 1 and 2 . The total cross section is then written as

$$
\sigma=\frac{1}{\operatorname{flux}} \prod_{j} \int \frac{\mathrm{d}^{3} p_{j}}{2 E_{j}(2 \pi)^{3}}(2 \pi)^{4} \delta^{4}\left(p_{1}+p_{2}-\sum_{j} p_{j}\right) \sum_{h_{f}} \sum_{h_{i}}\left|T_{i f}\right|^{2},
$$

where $T_{i f}$ is the helicity amplitude for the process $i \rightarrow f$. Initial helicities are averaged and the final ones are summed. This formula is used for the cross section in the GRACE/SUSY system. The cross section is given in the unit of pico-barn $(\mathrm{pb})$ in the kinematics library.

For the decay of a particle with momentum $p$, the total decay rate is given by

$$
\Gamma=\frac{1}{2 E} \prod_{j} \int \frac{\mathrm{d}^{3} p_{j}}{2 E_{j}(2 \pi)^{3}}(2 \pi)^{4} \delta^{4}\left(p-\sum_{j} p_{j}\right) \sum_{h_{f}} \sum_{h_{i}}\left|T_{i f}\right|^{2} .
$$

The independent integration variables expressed in terms of momenta and angles must be mapped onto those of the integration package BASES which are normalized as $0 \leq \mathrm{x}(\mathrm{i}) \leq 1$, where $\mathrm{i}$ runs from 1 to ndim, the dimension of the integration,

One should remember that the statistical factor for the identical particles is not generated automatically and should be supplied by hand. This is because this factor depends on the observables one is interested in. For the total cross section one should divide the result obtained by BASES by the factorial of the number of identical particles.

\section{C.2 Kinematics library}

GRACE/SUSY assumes the following two types of processes:

$$
\begin{array}{ll}
p_{1}+p_{2} \rightarrow p_{3}+p_{4}+\cdots & (2 \text {-body scattering) }, \\
p_{1} \rightarrow p_{2}+p_{3}+p_{4}+\cdots & \text { (decay of a particle) } .
\end{array}
$$

It should be noted that the particle assignment follows the order in which the particles are originally defined in in.prc.

The Lorentz invariant phase space element for a final $n$-body $(A \rightarrow 1+2+$ $3+\cdots+n)$ is defined by 


$$
\begin{aligned}
& d \tilde{\Gamma}_{n}=(2 \pi)^{4} \delta^{4}\left(\sum_{\text {in }} p-\sum_{\text {out }}^{n} p\right) \prod_{\text {out }}^{n} \frac{d^{3} p_{j}}{(2 \pi)^{3} 2 E_{j}} \equiv \frac{1}{(2 \pi)^{3 n-4}} d \Gamma_{n}, \\
& d \Gamma_{n}=\delta^{4}\left(\sum_{\text {in }} p-\sum_{\text {out }}^{n} p\right) \prod_{\text {out }}^{n} \frac{d^{3} p_{j}}{2 E_{j}}
\end{aligned}
$$

The following chain rule is useful $(0<k<n-1)$ :

$$
\begin{aligned}
d \Gamma_{n}(A \rightarrow 1+2 & +3+\cdots) \\
= & d \Gamma_{k+1}(A \rightarrow 1+\cdots+k+q) \frac{d Q^{2}}{2 \pi} \\
& \times d \Gamma_{n-k}(q \rightarrow(k+1)+(k+2)+\cdots),
\end{aligned}
$$

where $q^{2}=Q^{2}$.

When $p_{a}$ is in the center-of-mass system, i.e., $\mathbf{p}_{a}=\mathbf{0}$, and if $p_{a}=p_{b}+p_{c}$ we introduce the 2-body phase space

$$
d \Gamma_{\mathrm{CM}}(a ; b c) \equiv d \Gamma_{2}=\delta^{4}\left(p_{a}-p_{b}-p_{c}\right) \frac{d^{3} p_{b}}{2 E_{b}} \frac{d^{3} p_{c}}{2 E_{c}}
$$

and write it by angular variables:

$$
d \Gamma_{\mathrm{CM}}(a ; b c)=\frac{\beta(a ; b c)}{8} d \Omega_{\mathrm{CM}}(a ; b c)=\frac{\beta(a ; b c)}{8} d \cos \theta_{b,(b c)} d \phi_{b,(b c)}
$$

Here $\beta$ is given by

$$
\begin{aligned}
\beta(a ; b c) & =\frac{2 P}{E_{a}} \\
& =\frac{1}{E_{a}} \sqrt{\left(E_{a}+m_{b}+m_{c}\right)\left(E_{a}-m_{b}-m_{c}\right)\left(E_{a}+m_{b}-m_{c}\right)\left(E_{a}-m_{b}+m_{c}\right)} .
\end{aligned}
$$

The subscript $(b c)$ of the angles indicates that $\theta_{b}, \phi_{b}$ are defined in the centerof-mass frame. Angles in the laboratory frame have no subscript. 
Kinematics routines prepared in the library are summarized in Table C.2.

\begin{tabular}{|c|c|}
\hline code number & contents \\
\hline 1201 & 1-body $\rightarrow 2$ body decay \\
\hline 1301 & $\begin{array}{l}\text { 1-body } \rightarrow 3 \text { body decay } \\
\text { Sequential decay } 1 \rightarrow 2+(3+4) \rightarrow 2+3+4 \text { can be treated. }\end{array}$ \\
\hline 2201 & $\begin{array}{l}2 \text {-body } \rightarrow 2 \text { body in CM frame } \\
t \text { - and } u \text {-channel singularities can be treated. }\end{array}$ \\
\hline 2301 & $\begin{array}{l}\text { 2-body } \rightarrow 3 \text { body in CM frame } \\
\text { Sequential decay type } 1+2 \rightarrow 3+(4+5) \rightarrow 3+4+5 \text {. } \\
\text { Resonance on particles } 4 \text { and } 5 \text { can be treated. }\end{array}$ \\
\hline 2302 & $\begin{array}{l}\text { 2-body } \rightarrow 3 \text { body in } \mathrm{CM} \text { frame } \\
\text { Radiative processes } 1+2 \rightarrow 3(\gamma)+4+5 \\
\text { both initial and final radiation can be treated. }\end{array}$ \\
\hline 2303 & $\begin{array}{l}2 \text {-body } \rightarrow 3 \text { body in CM frame } \\
\text { Double-radiative processes } 1+2 \rightarrow 3(\gamma)+4(\gamma)+5\end{array}$ \\
\hline 2304 & $\begin{array}{l}\text { 2-body } \rightarrow 3 \text { body in CM frame } \\
\text { Three photon processes } 1+2 \rightarrow 3(\gamma)+4(\gamma)+5(\gamma)\end{array}$ \\
\hline 2401 & $\begin{array}{l}\text { 2-body } \rightarrow 4 \text { body in CM frame, a pair of sequential } \\
\text { decay type } 1+2 \rightarrow(3+4)+(5+6) \rightarrow 3+4+5+6 \\
t \text {-channel singularity can be treated. } \\
\text { 2-body } \rightarrow 4 \text { body in CM frame, } \\
\text { 'fusion' type } 1+2 \rightarrow(3+A)+(4+B) ; A+B \rightarrow 5+6\end{array}$ \\
\hline
\end{tabular}

Table C.2 Kinematics library prepared in GRACE/SUSY system.

\section{References}

[1] For review see, for example, P. Fayet and S. Ferrara, Phys. Rep. 32 (19877) 249; H. Nilles, Phys.Rep. 110 (1984) 1; H. Haber and G. Kane, Phys. Rep. 117 (1985) 75 . 
[2] T.Ishikawa et al., KEK Report 92-19, 1993, The GRACE manual v1.0; F. Yuasa et al., Prog. Theor. Phys. Suppl. 138 (2000) 18, hep-ph/0007053.

[3] T. Kon, "Automatic calculation of SUSY amplitudes" in Les Arcs 1995, Electroweak interactions, unified theories, pp. 287-292.

[4] H. Tanaka, M. Kuroda, T. Kaneko, M. Jimbo and T. Kon, Nucl. Instrum. Meth. A 389, 295 (1997).

[5] J. Fujimoto et al., Comput. Phys. Commun. 111 (1998) 185.

[6] J. Rosiek, Phys. Rev. D41 (1990) 3463, erratum KA-TP-8-1995, hep$\mathrm{ph} / 9511250]$.

[7] K. Hikasa, SUSY manuscript version July 5, 1995 unpublished.

[8] M. Kuroda, KEK CP-080, 1999, hep-ph/9902340.

[9] G. Farrar and P. Fayet, Phys. Lett B76 (1976) 575;

$\mathrm{R}$. Barbier et al., Report of the GDR working group on the R-parity violation, hep-ph/9810232.

[10] J.F. Gunion and H.E. Haber, Nucl. Phys. B272 (1986) 1.

[11] A. Arhrib and G. Moultaka, PM/97-25, Nucl. Phys. B558 (1999) 3.

[12] A. Semenov, LAPTH-884/01.

[13] F. Boudjema and A. Semenov, LAPTH-880/01, hep-ph/0201219.

[14] M. Kuroda, KEK CP-057, 1997, hep-ph/9707446.

[15] T. Kaneko and H. Tanaka in Proc. in the second workshop on JLC, ed. S. Kawabata, 1991 p.250.

[16] A. Denner et al., Nucl. Phys. B387 (1992) 467.

[17] G. Bélanger et al., "micrOMEGAs: a program for calculating the relic density of neutralinos" in The Second Symposium on Computational Particle Physics, Tokyo 2001, to be published.

[18] E.E Boos et al. in New Techniques in Physics Research, ed. D. Perret-GAllix and W. Wojcik, Ed. CNRS, Paris, 1990, p.573;

P.A. Baikov et al., Proc. of Xth Workshop on High Energy Physics and Quantum Field theory (QFTHEP-95), ed. B. Levtchenko and V. Savrin, Moscow 1996, p101, hep-ph/9701412];

E.E. Boos, M.N. Dubinin, V.A. Ilyin, A.E. Pukhov and V.I. Savrin, "CompHEP: Specialized package for automatic calculations of elementary particle decays and collisions" in Korean Physical Society meeting, October 21, 1994. hep$\mathrm{ph} / 9503280$.

[19] V. Lafage, T. Ishikawa, T. Kaneko, T. Kon, Y. Kurihara and H. Tanaka, Int. J. Mod. Phys. A 14, (1999) 5075, hep-ph/9810504. 
[20] G. Bélanger et al., "Achievements beyond the standard model," in The First Symposium on Computational Particle Physics, KEK Proceedings 99-23, Tokyo 1998 pp.35-43.

[21] G. Bélanger, F. Boudjema, T. Kon and V. Lafage, Eur. Phys. J. C 9 (1999) 511, hep-ph/9811334.

[22] G. Bélanger, F. Boudjema, T. Kon and V. Lafage, Eur. Phys. J. C 12, (2000) 323, hep-ph/9907207.

[23] Y. Kurihara, V. Lafage and T. Kon, "A scalar-charm production and its decay at HERA" in Workshop on Monte Carlo Generators for HERA Physics (Plenary Starting Meeting), Hamburg, Germany, 27-30 April, 1998.

[24] T. Matsushita, T.. Abe, S. Kitamura, T. Kobayashi and T. Kon, "Stop and sbottom production at HERA" in Workshop on Monte Carlo Generators for HERA Physics (Plenary Starting Meeting), Hamburg, Germany, 27-30 April, 1998.

[25] S. Kitamura, T. Kon and T. Kobayashi, Mod. Phys. Lett. A 16, (2001) 947, hep-ph/0012166.

[26] T. Kon, T. Kobayashi, S. Kitamura and T. Iimura, Phys. Lett. B 494, (2000) 280, hep-ph/0007200.

[27] I. Watanabe et al., Gamma gamma collider as an option of JLC, KEKREPORT-97-17.

[28] T. Kon, Nucl. Instrum. Meth. A 472 (2001) 239.

[29] B. Badelek et al. [ECFA/DESY Photon Collider Working Group Collaboration], TESLA Technical Design Report, Part VI, Chapter 1: Photon collider at TESLA, hep-ex/0108012].

[30] K. Abe et al. [ACFA Linear Collider Working Group Collaboration], Particle physics experiments at $J L C$, hep-ph/0109166.

[31] S. Abdullin et al., The SUSY working group: Summary report, hep$\mathrm{ph} / 0005142$.

[32] M. Besançon and E. Dudas et al., Report of the GDR working group on the $R$-parity violation, hep-ph/9810232, related references will be found therein.

[33] V. Lafage et al., Int. J. Mod. Phys. A 14 (1999) 5075;

V. Lafage, http://minami-home.kek.jp/lafage/.

[34] S. Heinemeyer et al., Phys. Rev. D58 (1998) 091701, hep-ph/9803277; Phys. Lett., B440 (1998) 296, [hep-ph/9807423]; Eur. Phys. J. C9 (1999) 343, hepph/9812472]; Phys.Lett. B455 (1999) 179, hep-ph/9903404.

[35] Minami-Tateya Collaboration, http://minami-home.kek.jp/gracesusy.html

[36] Y. Kurihara, in preparation. 